\title{
Spread F - an old equatorial aeronomy problem finally resolved?
}

\author{
R. F. Woodman \\ Radio Observatorio de Jicamarca, Instituto Geofísico del Perú, Lima, Peru
}

Received: 28 October 2008 - Revised: 25 March 2009 - Accepted: 27 March 2009 - Published: 4 May 2009

\begin{abstract}
One of the oldest scientific topics in Equatorial Aeronomy is related to Spread-F. It includes all our efforts to understand the physical mechanisms responsible for the existence of ionospheric F-region irregularities, the spread of the traces in a night-time equatorial ionogram - hence its name - and all other manifestations of the same. It was observed for the first time as an abnormal ionogram in Huancayo, about 70 years ago. But only recently are we coming to understand the physical mechanisms responsible for its occurrence and its capricious day to day variability. Several additional techniques have been used to reveal the spatial and temporal characteristics of the F-region irregularities responsible for the phenomenon. Among them we have, in chronological order, radio star scintillations, trans-equatorial radio propagation, satellite scintillations, radar backscatter, satellite and rocket in situ measurements, airglow, total electron content techniques using the propagation of satellite radio signals and, recently, radar imaging techniques. Theoretical efforts are as old as the observations. Nevertheless, 32 years after their discovery, Jicamarca radar observations showed that none of the theories that had been put forward could explain them completely. The observations showed that irregularities were detected at altitudes that were stable according to the mechanisms proposed. A breakthrough came a few years later, again from Jicamarca, by showing that some of the "stable" regions had become unstable by the non-linear propagation of the irregularities from the unstable to the stable region of the ionosphere in the form of bubbles of low density plasma. A problem remained, however; the primary instability mechanism proposed, an extended (generalized) Rayleigh-Taylor instability, was too slow to explain the rapid development seen by the observations. Gravity waves in the neutral background have been proposed as a seeding mecha-
\end{abstract}

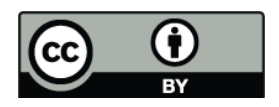

Correspondence to: R. F. Woodman (ronw@geo.igp.gob.pe) nism to form irregularities from which the instability would grow, but the former are difficult to observe as a controlling parameter. Their actual role still needs to be determined. More recently, radar observations again have shown the existence of horizontal plasma drift velocities counter streaming the neutral wind at the steep bottom of the F-region which produces a fast growing instability from which a generalized Rayleigh-Taylor instability can grow. The mechanisms proposed would explain the rapid development of the large and medium scale irregularities that have been observed, including some seen only by radars. Nevertheless, a proper quantitative theoretical mechanism that would explain how these irregularities break into the very important meter scale ones, responsible for the radar echoes, needs to be developed. This paper makes a selective historical review of the observations and proposed theories since the phenomenon was discovered to our current understanding.

Keywords. Ionosphere (Equatorial ionosphere; Ionospheric irregularities) - Space plasma physics (Waves and instabilities)

\section{Introduction}

It is for me an honour to have been asked to contribute with a tutorial review to this special issue dedicated to the memory of my good friend and colleague Tor Hagfors, whom I consider to be the best radio scientist I have ever known. The subject choosen for my review is appropriate, considering the importance of the paper we co-authored together describing a technique to measure ionospheric drift (Woodman and Hagfors, 1969), a crucial parameter for the understanding of the physical mechanisms responsible for Spread F.

In this review I will take an historical approach, starting with the first observations made in Huancayo (Booker and Wells, 1938). But, considering its very long 70 year history

Published by Copernicus Publications on behalf of the European Geosciences Union. 
H. G. BOOKER AND H.W.WELLS

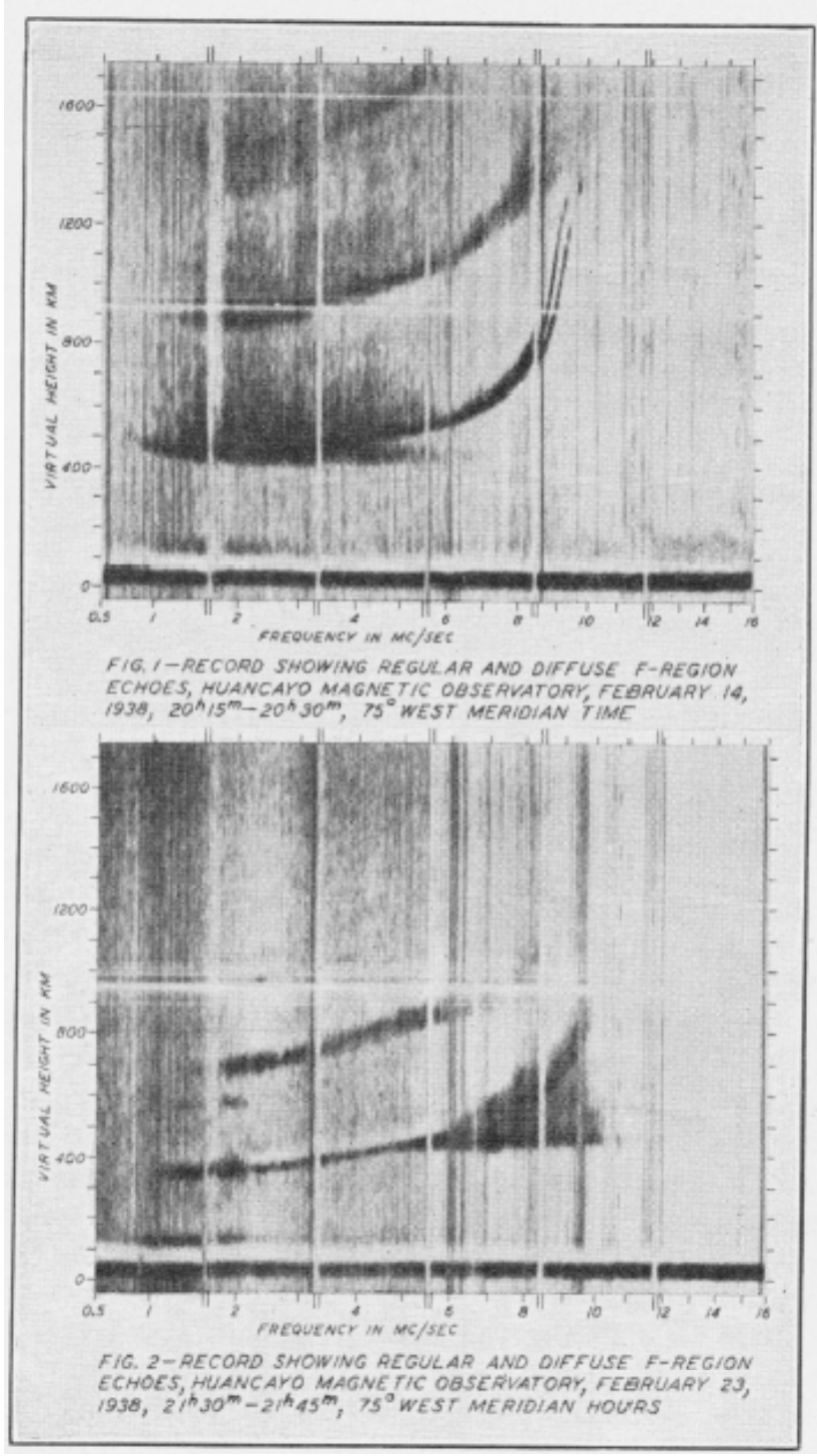

Fig. 1. First published ionogram showing Spread F (after Booker and Wells, 1938).

and the limited space allocated, I have to be very selective and limit myself to those contributions that I consider to be key stepping stones in the progress toward understanding the nature of the phenomenon and the physical mechanisms responsible for its existence. Note the "I consider" in the previous sentence; it implicitly reveals a subjective and biased position. Many important papers on the subject will not be mentioned, and I apologize for my omissions with the hope that they will be indirectly acknowledged in the references of those that I do mention, especially those that have the nature of a review. The bias will be influenced by my own work and by my familiarity with the contributions made by Peruvian observatories at Huancayo, Ancon and Jicamarca.

\section{Early observations}

What was originally observed by Booker and Wells (1938) were "diffuse echoes" from the F-region obtained in Huancayo by the recently installed "automatic multifrequency technique of vertical radio sounding," later known simply as an ionosonde. The ionogram traces, rather than showing a thin line corresponding to the virtual height of the reflecting altitude, as the ionosonde frequency was changed, showed instead a range of virtual heights as if the echoing region were spread over a range of altitudes (range spread). At times, the spread showed only at the high frequency end and looked more like a spread in frequency for a given virtual height (frequency spread). For many years the ionograms were scaled and parameterized before being distributed to the interested community. Among the parameters was foF2, the critical (plasma) frequency corresponding to the maximum density of the F-region. When the critical frequency could not be read because of spread appearance of the echoes, the term "Spread F" was used to indicate the reason for its omission. Although spread echoes appear at other latitudes as well, we are concerned here with Equatorial Spread F. Figure 1 shows the first ionogram published showing such conditions.

Booker and Wells (1938) interpreted correctly that the diffusive nature of the echoes was produced by radio scattering from electron density fluctuations. The existence of such fluctuations has been detected, as we shall soon see, by other instruments and techniques. Now, whenever such conditions exist in the F-region, we often refer to the phenomenon as Equatorial Spread F, or ESF for short. A more appropriate name for this condition is Equatorial F-Region Irregularities or Equatorial F-Region Field Aligned Irregularities (FAIs), even though different instruments are sensitive to different length scales of the irregularities, and so simultaneous observations with different techniques do not necessarily correlate. Nevertheless, we will use the terms as synonymous as is frequently done in the literature.

Chronologically the second technique sensitive to the existence of FAIs was the scintillations of radio stars. FAIs also occur at non-equatorial latitudes, and it was there that radio scintillation was first used for their study (e.g., Booker, 1956).

With the advent of the space age and the existence of satellites, a much more powerful radio scintillation technique became available. Satellite scintillation techniques provided, at any given latitude and longitude, more sources at any given time of day than did the available radio stars. The first use of satellite scintillations (Woodman, 1960) has an interesting anecdote. Shortly after Sputnik and before the first US satellite was launched, the Minitrack, a network of satellite tracking and receiving stations built along the $75^{\circ} \mathrm{W}$ meridian, was operational. One of the stations was located in Ancon, near Lima, Peru, about $70 \mathrm{~km}$ north of the magnetic equator. Like the others, it had an interferometer to precisely 


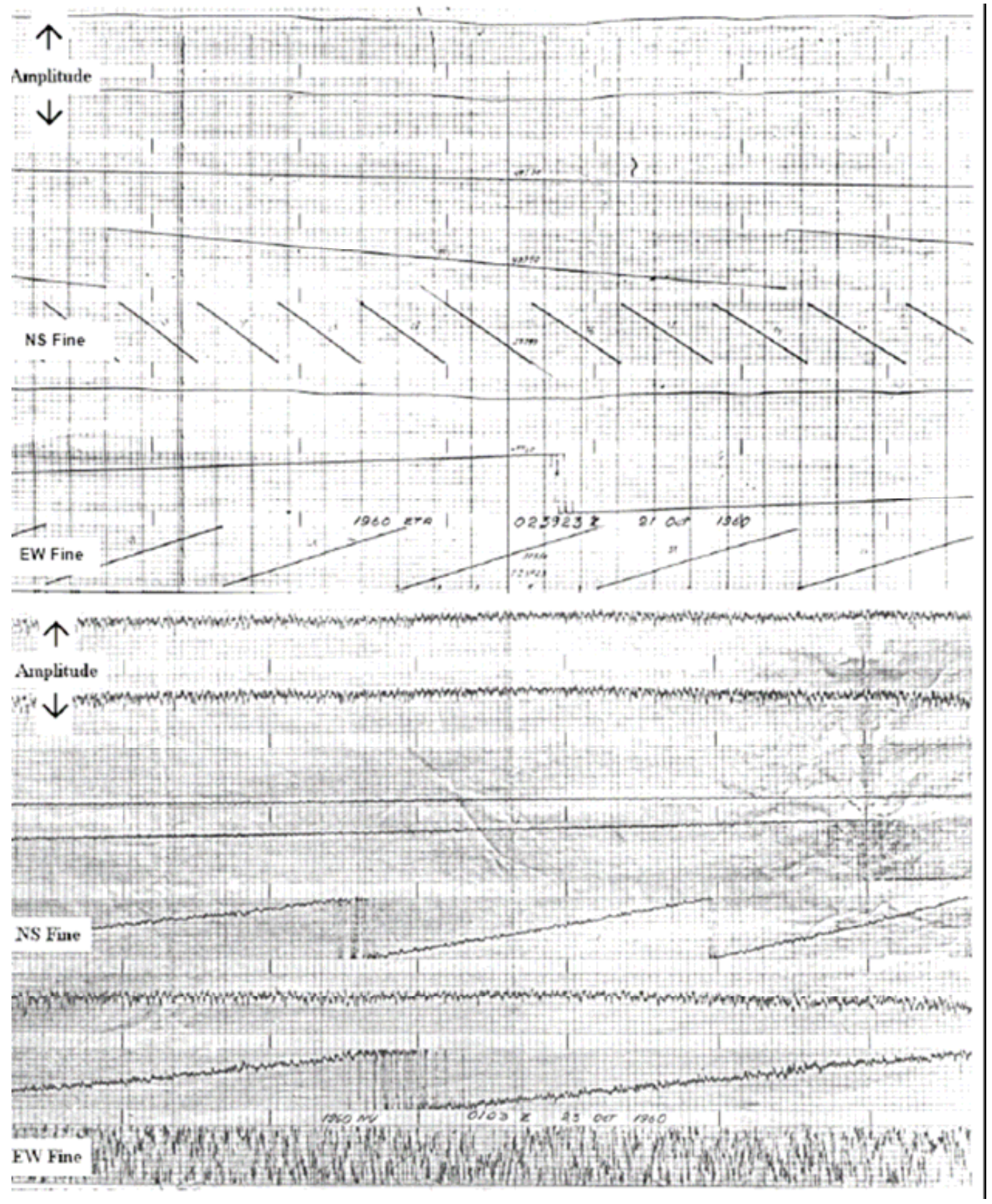

Fig. 2. Interferometric phase records taken in 1960 at Ancon, Peru, of two satellite passes: (top panel) during normal conditions, (bottom panel) during Spread F conditions. There are four record channels of interest on each (out of the eight shown), the top 2, showing the amplitude (log scale), and the interferometric phase ( 0 to $2 \pi$, corresponding to $1^{\circ}$ of physical angle) for the NS and EW Fine base lines. Notice the strong phase scintillations in the EW channel in panel b) while the NS channel is undisturbed. Both fine base-line amplitudes (top channels) showed amplitude scintillations (adapted from Woodman, 1960).

determine the satellite position (tracking) and subsequently its orbit. When the tracking of the first American satellites began, it was realized that the station had a "problem": in certain satellite passes, usually at night, one of the channels, the East-West Fine channel, would go wild. It became completely noisy, as shown in Fig. 2. The corresponding North South channel was undisturbed. The engineers and techni- cians, I among them, had an assignment: to find out what was wrong with the EW channel. The problem even had a name at the Satellite Tracking Division, NASA's Goddard Space Flight Center, where it was called the Cunningham Effect, after the name of the Ancon station director.

After many months on this effort, I was fortunate to run into the papers by Booker (1958) and Koster and Wright 


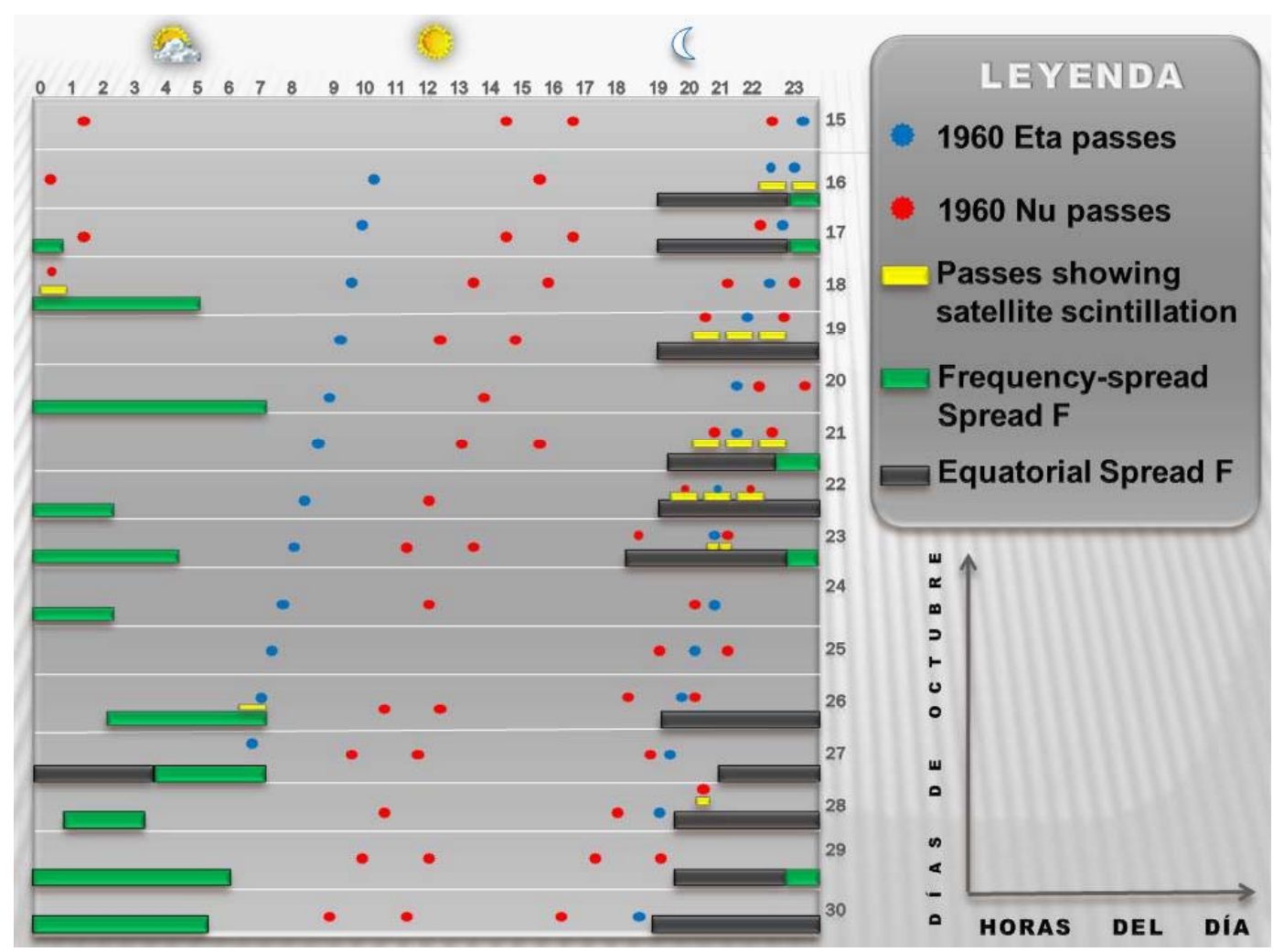

Fig. 3. Graphic showing the state of Spread F for different days, different times of the day and the effect it had on two satellites, 1960 Eta and $1960 \mathrm{Nu}$. Those passes that have a yellow underline showed satellite scintillation. Notice the good correlation between scintillation and Spread F (adapted from Woodman, 1960).

(1960) that had used radio star scintillations to study FAIs, the latter at the equator, and in relation to Spread F. A correlation study with Spread F, as observed with the Huancayo ionosonde, was carried out showing a direct relationship, as shown in Fig. 3 (Woodman, 1960). The undisturbed NS interferometer traces were explained by postulating irregularities that were very elongated in the NS direction. Although physically expected, it was the first time that such an elongation was directly observed. Not only was the "equipment problem" solved, but a relatively simple technique was born for the study of the irregularities responsible for Spread F. It required only an antenna and a receiver tuned to the beacon or telemetry frequency used by the satellites. It did not have the temporal constraints of the radio stars and did not required large antennas. The potential of the technique increased with the increase in the number of satellites and with the number and range of frequencies of their transmitters.

It is interesting to note that the same observations published by Woodman (1960), were also presented at the first ISEA meeting in Huaychulo, Peru, in 1962.

Both ionosonde and scintillation receivers have been used at different locations to determine the temporal, seasonal and geographical distribution of Spread F for different solar activity conditions. A large amount of work has been invested in this effort (see for instance Aarons, 1977; Basu and Basu, 1981, 1985; Abdu et al., 1998, and references therein). We will not reproduce here all the details of their findings. It is sufficient to say that it is a nighttime phenomenon, occurring mainly before midnight, except for magnetically disturbed conditions, and that it occurs in a belt $30-40^{\circ}$ wide, centred on the magnetic equator. A good example of this type of statistics is found in Fig. 4. Their seasonal behavior depends on longitude, a dependence that was explained by Tsunoda (1985) in terms of the geographical latitude of the magnetic equator and the inclination of the day-night terminator with respect to the declination of the magnetic field at each particular longitude. This explanation also gives an important clue on the importance of the transverse conductivity at the foot of the magnetic field lines at the time of the onset of the irregularities.

Satellite scintillations, besides being used as a powerful and simple tool to study FAIs, are also a reason of concern. They affect the quality of the signals used for application satellites. They deteriorate the quality of the signals of telecommunication satellites as well as those of the telemetry of all the others, like weather, remote sensing and scientific satellites. In the case of radar satellites used as altimeters or to obtain radar images, they affect the quality of the 


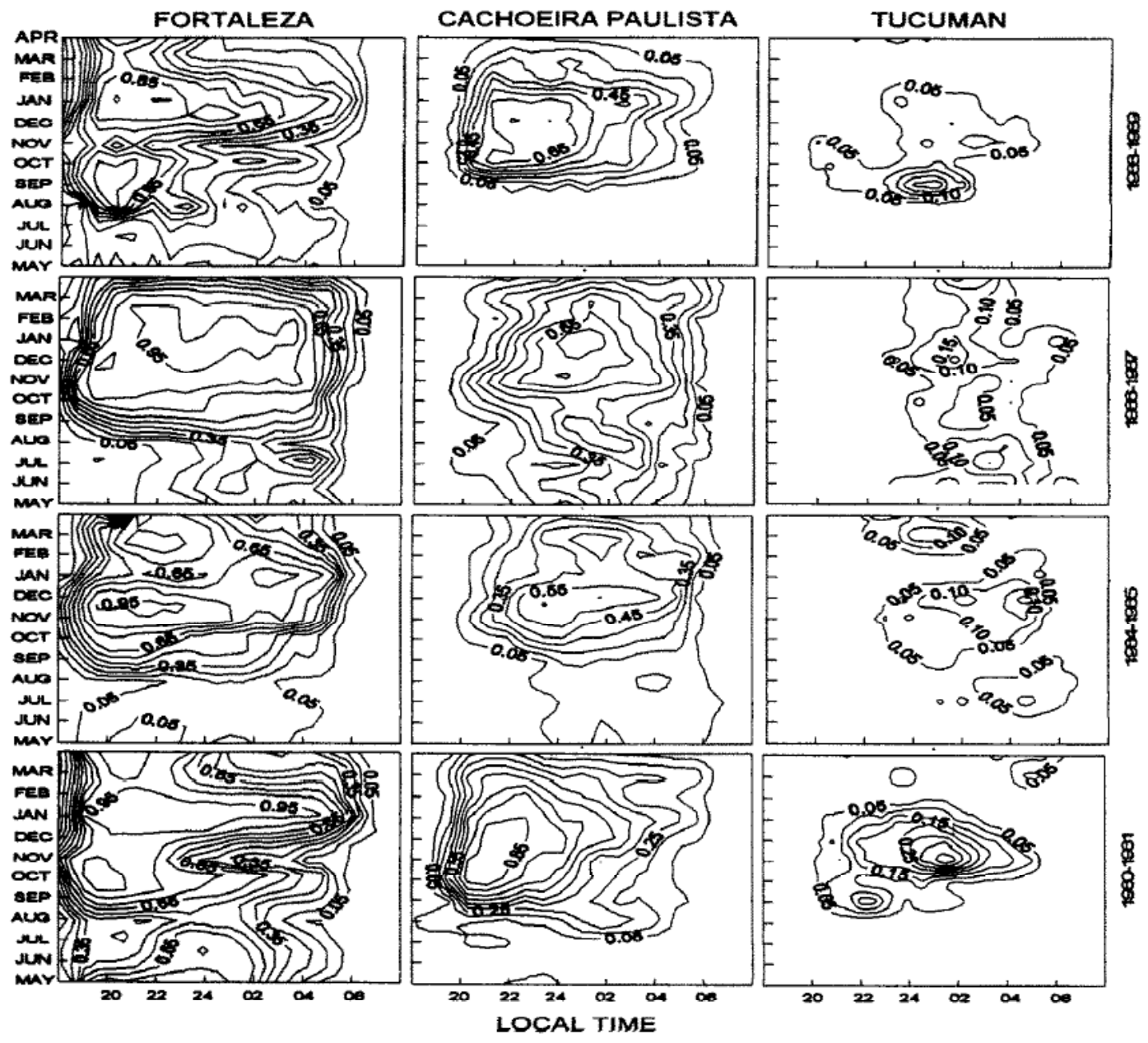

Fig. 4. Frequency of occurrence of Spread F for the Eastern South American sector (after Abdu et al., 1998).

final product both ways, on transmission and reception. They also affect the accuracy of high precision location using GPS satellites.

An additional diagnostic for Spread F is the forward scatter technique (see for instance, Cohen and Bowles, 1963). A transmitter is placed south or north of the magnetic equator and a receiver in the opposite hemisphere. The antennas used have sufficient gain to define a common volume at a particular F-region height that is known to have FAIs. On reception, the signal power is recorded with sufficient temporal resolution to determine the fading rate. The technique was used by Cohen and Bowles (1963) to study the seasonal behavior of Spread F in Peru during the IGY. The seasonal statistics of the intensity of the scattering conformed well with what was known from ionosonde observations.

Spread F shows considerable seasonal as well as day to day variability within a particular season. Ionosondes have been used to study this variability, which is still not completely understood. A significant correlation has been found with the altitude of $h^{\prime}$, the virtual height of the bottom of the
F-region, and with its rate of change. What has become clear is that Spread F prefers high values of $h^{\prime}$ and a recent past history with positive rates of change (e.g., Rastogi, 1978, and references therein). We will come back to discuss this variability when we discuss current research efforts.

An important historical observation was reported by Röttger (1973). In a long distance HF trans-equatorial propagation experiment (Lindau, Germany to Tsumeb, South Africa), he noticed multiple arrival times of the transmitted signal. Besides the arrival time corresponding to the great circle path reflected from the F-region, other arrival times were spread over a range of delays but grouped discretely in a periodic fashion (see Fig. 5). The delayed signals were interpreted as coming from scattering from discrete regions of F-region FAIs east and west of the great circle joining the transmitter and receiver sites. The periodicity of the delays triggered many theories involving gravity waves and its relationship with Spread F. One of them, still much in vogue, used gravity waves to modulate the background electron density to provide a seeding for plasma instabilities that would 


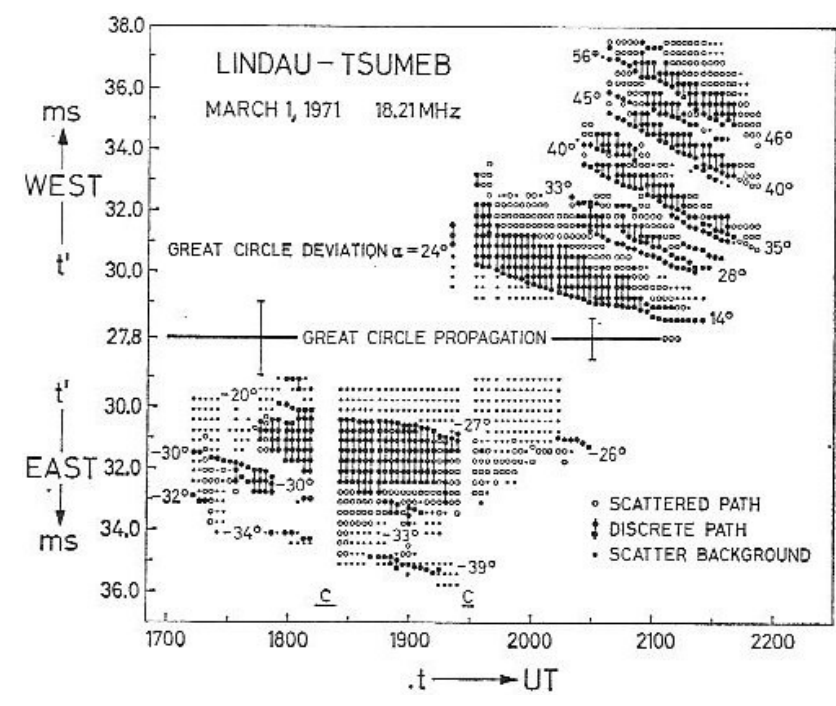

Fig. 5. Propagation delay characteristics of a trans-equatorial link between Lindau and Tsuneb (after Roettger, 1973). Note the diffuse nature of the delays clustered in distinct groups with, delays larger than the one corresponding to the great circle. The degrees label on each cluster correspond to the deviation angle of the propagation path with respect to the great circle.

allow them to grow to the very large fluctuations responsible for Spread F in a relative short time (see Kelley et al., 1981, and references therein).

\section{Jicamarca's early contributions}

The Jicamarca Radar Observatory (JRO) became operational in the early 1960s (Bowles et al., 1962). It had been built to obtain electron densities and temperatures as well as ion composition using the Incoherent Scatter technique. It was the first of its kind to become operational worldwide, and it is still the most sensitive radar at equatorial latitudes. From the very beginning, it was realized that Spread F conditions produced huge coherent echoes, but the relationship between Spread F and radar echoes was not studied in detail and published until later (Rastogi, 1978). Rastogi (1978) also established that the frequency type of Spread F did not produce strong echoes in the radar, at least when the antenna was offperpendicular measuring IS densities.

For many years, Spread F was considered a nuisance, since it prevented the measurements the instrument was designed to make, and little effort was dedicated to studying it in a systematic way. The advantage of the Jicamarca radar over previous techniques was that it could detect with precision the altitudes at which the irregularities exist and it did not have the altitude limitations of ground based ionosondes; it could detect their presence way above the density maximum. The radar is sensitive only to vertically propagating (vertical k-vector) 3-m irregularities (half the wave length corre-

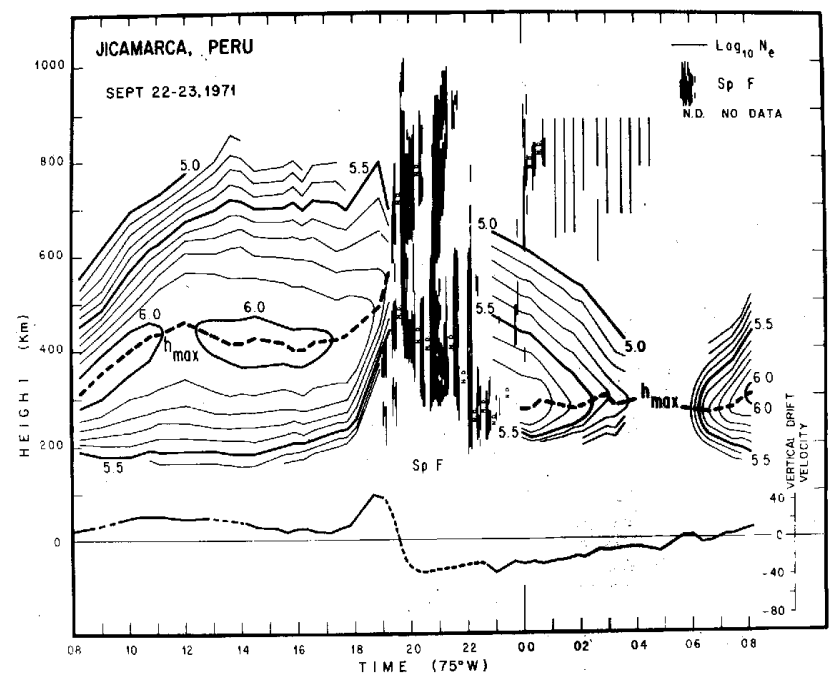

Fig. 6. Plot of electron density, vertical drift and range-time occurrence of Spread F. Although taken from Woodman and La Hoz (1976), it illustrates the conclusions of Farley et al. (1970): None of the current theories existing then could explain the simultaneous presence of irregularities at positive and negative electron density gradients or positive and negative eastward electric fields.

sponding to the radar frequency). Its relationship to the larger irregularities responsible for Spread $\mathrm{F}$ and satellite scintillation implicitly assumes that they result from a breakdown of the larger irregularities (e.g. Haerendel, 1973).

The first systematic study of Spread F using Jicamarca's potential came later in a paper by Farley et al. (1970). The potential of the Jicamarca radar had been greatly enhanced with the development of a technique to measure vertical drifts using incoherent scatter (Woodman and Hagfors, 1969). The Jicamarca radar was then able to measure the electron density and the zonal electric field (responsible for the vertical drift) of the background ionosphere, two of the most important state parameters related to its possible instability. The importance of the Farley et al. (1970) paper comes from a negative conclusion: none of the theories that had been put forward to explain the physical mechanisms responsible for the occurrence of Spread F could explain the observations. Two of the theories had strong support, in fact they could supplement each other. Both required a gradient in electron density. One of them was first proposed by Dungey (1956). He suggested that the irregularities were a consequence of a gravitational instability. Indeed, the steep gradient at the bottom of the F-region under the effects of gravity is unstable. It is clear that the opposite gradient at the top is stable. The other theory originated by Martyn (1959), claimed that the underside of the F-region was unstable under the influence of an electromagnetic upward drift. Although not discussed by Martyn, it is clear that if one reverses the sign of either the gradient or the vertical drift, the situation is stabilizing. The arguments given by Farley et al. (1970) are 


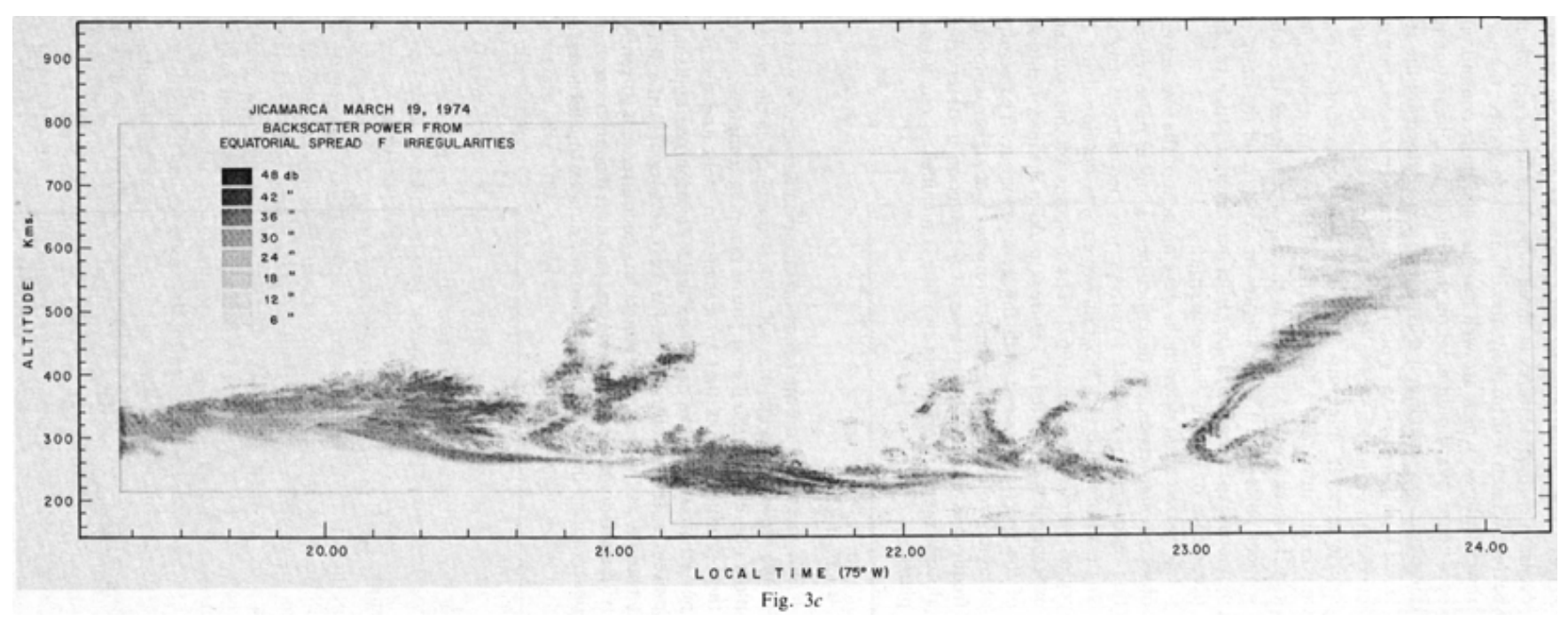

Fig. 7. Range-time-intensity plot of backscattered echoes from Spread F irregularities. The plume starting at $2300 \mathrm{~h}$ and $300 \mathrm{~km}$ altitude connected with the $600 \mathrm{~km}$ irregularities an hour later was the clue to explain the irregularities at gravitational stable altitudes (after Woodman and La Hoz, 1976).

well illustrated by Fig. 6, taken from a later paper by Woodman and La Hoz (1976). There, one can notice that Spread $\mathrm{F}$ occurs either at the bottom or the top of the F-region when the vertical drifts are positive or negative, i.e., the Spread is present when either one or both candidate mechanisms present stable conditions.

Haerendel (1973) in an unpublished manuscript proposed that the largest scales of Spread F were produced by a gravitational instability as proposed by Dungey (1956) and that the smaller scales resulted from a cascade of instabilities all the way down to the meter scales responsible for the radar coherent echoes. Furthermore, he extended the altitude range of the primary instability into the local negative gradients on top of the F-region peak. He postulated that a magnetic flux tube, because of the large conductivities along the magnetic field lines, behaved as an entity, and what was important was not the local vertical electron density gradient but rather the gradient of the integrated density, integrated along the field line. Nevertheless, this extension in altitude still did not explain the existence of irregularities at higher altitudes where even the integrated density gradients had negative stable values.

\section{Plumes and bubbles}

In the 1970s, there was a rapid development of (then) powerful computers at affordable prices. This made it possible to process power and spectral information from the Jicamarca radar echoes at multiple altitudes in real time. Among the new products, the possibility of producing two-dimensional maps of Spread F echo power as a function of altitude and time allowed for a significant breakthrough in our under- standing of the primary instabilities responsible for spread $\mathrm{F}$. The occurrence of a plume like feature in one of these maps (see Fig. 7) led Woodman and La Hoz (1976) to postulate a mechanism to produce irregularities at the locally stable topside of the F-region. They postulated that a large (in size) perturbation in the gravitationally unstable bottom side of the F-region would grow nonlinearly into a bubble, in a way not much different than in the case of a Rayleigh-Taylor instability in a neutral fluid, when a heavier fluid rests on top of a lighter one. The bubble will rise by the effect of buoyancy and will continue to do so even in regions with gravitationally stable gradients, provided the density entrapped in the bubble is lower than that of the surroundings. It should be recalled that the plasma density below the bottom of the F-region is lower than the density at $1000 \mathrm{~km}$ or more. The mechanism was illustrated by means of Fig. 8a, where a fluid of a given density rests on top of a lighter one but is under a fluid of intermediate density. The sequence shows how a perturbation in the lower interface would propagate to the top fluid, despite the stability of the top interface. The feature in the power map shown in Fig. 7 that gave the clue to Woodman and La Hoz (1976), is the "plume" that started in the bottom side of the F-region at around 23:00 h LT and reached $600 \mathrm{~km}$ around 24:00 LT. It was the continuity of the trace that led them to their interpretation.

Other maps in the same reference give further support to the same explanation. The problem of understanding the presence of FAIs at the stable top of the ionosphere was solved. The qualitative theory of Woodman and La Hoz (1976) was soon supported by numerical simulations (Scannapieco and Ossakow, 1976) (this sequence of events is sometimes inverted in review papers and historical 

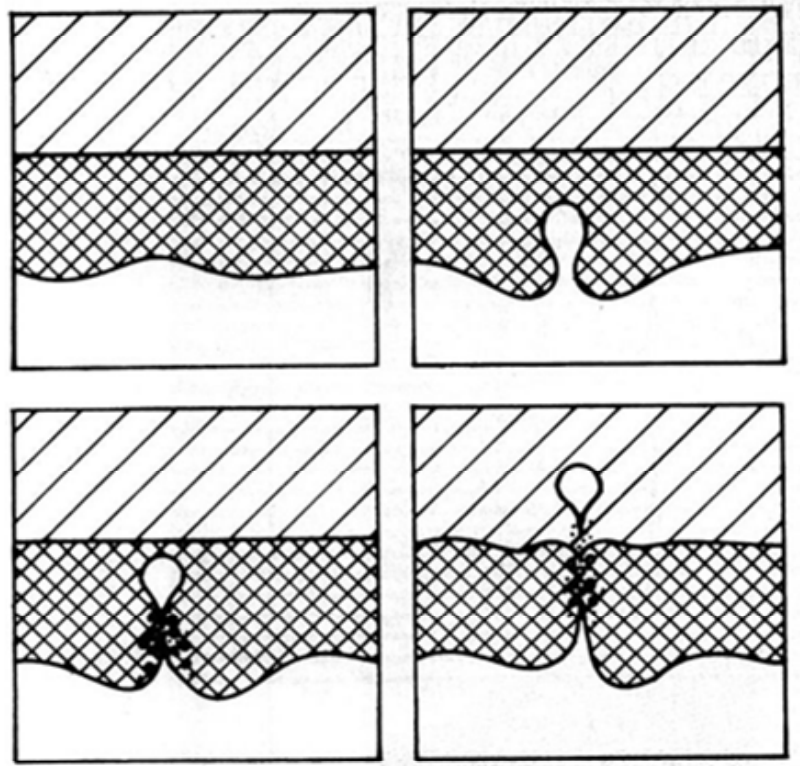
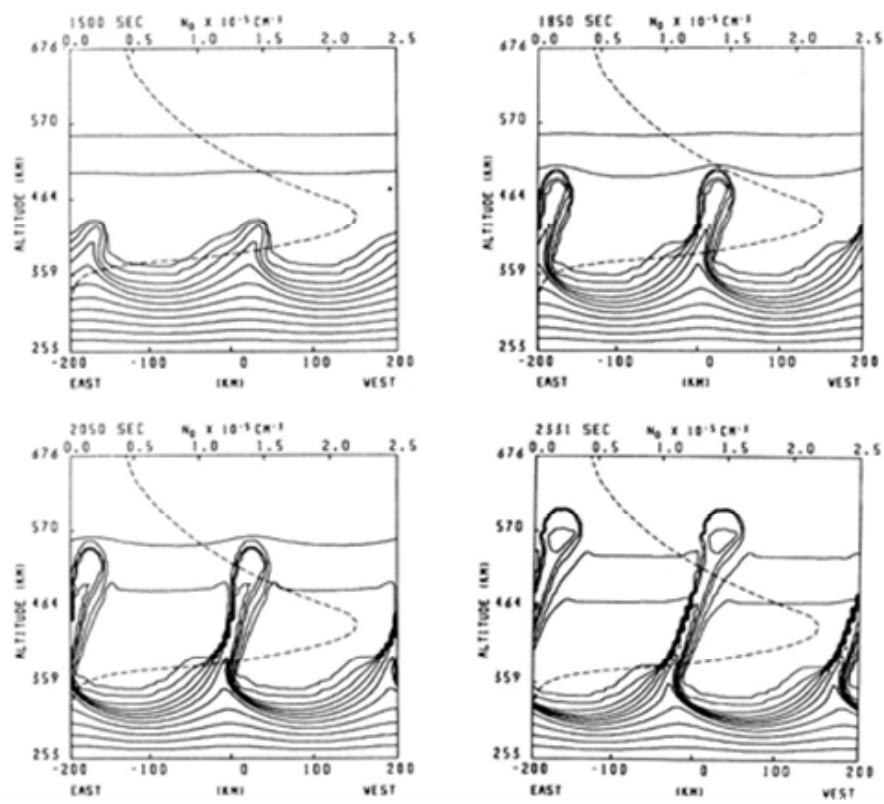

Fig. 8. The left panel shows a Woodman and La Hoz (1976) sketch of how a low density bubble propagates to the stable top provided that the densities there are higher than those in the lower third. The right panel shows the result of a computer simulation by Zalesak et al. (1982) of the same. The westward tilt reproduces the tilt of the plumes and is produced by a eastward neutral wind, also in accord with the Woodman and La Hoz (1976) explanation.

introductions). The idea of a bubble "floating" to the top was first presented by Woodman at the 1975 Gordon Conference on Space Plasma Physics where it was suggested that the NRL code used to explain striations in Barium cloud releases could simulate the bubble formation. Refinements were made to the original simulation (see Ossakow, 1981, for a review and references) and Fig. 8b shows later results obtained numerically by Zalesak et al. (1982), for a $200 \mathrm{~km}$ wave perturbation. The similarity with Fig. $8 \mathrm{a}$ is evident. The tilt of the plume was not shown in the Woodman and La Hoz (1976) sketch, but it was discussed and explained in the text in terms of a vertical electric field in the frame of the neutrals, i.e. a differential horizontal drift between the plasma and the neutrals. Such an electric field was included in the later numerical simulation of Zalesak et al. (1982).

The effect of the EW electric field responsible for the uplift of the F-region, shortly before Spread F appears, helps to make any horizontal wave perturbation more unstable. In fact, the frictional force produced by the relative vertical velocity of the neutrals $(W)$, and the gravitational force proportional to gravity $(g)$, combine linearly as a single term in the momentum equation and in the linear growth rate. The theory involving both sources of instability is now referred to as Generalized Rayleigh-Taylor (GRT) instability (e.g. Kelley, 1989). Of course, what is an electric field and what is a relative plasma drift velocity with respect to the neutrals depends on the choice of the frame of reference. They are all interchange instabilities, and they are often referred to under that name. The name comes from the fact that density fluctuations in a plasma, being incompressible in the plane transverse to the magnetic field, can come about only by transporting low density plasma into a higher density region and high density into a lower density one, interchanging positions.

The Range-Time-Intensity (RTI) plots shown in Woodman and La Hoz (1976), besides showing the plumes propagating to the top side of the ionosphere, as we have already discussed, showed that, on many occasions, the radar would see strong echoes that were confined only to the bottom side of the F-region. The thickness of these echoes was often reduced, especially during their early appearance, to one or a few range gates $(5 \mathrm{~km}$ radar pulse length and sampling), at the very bottom of the F-region. On occasions, the valley region below the F-region would also go unstable. Woodman and La Hoz (1976) proposed that the different manifestations should be classified into different types and referred to them as Top Side, Bottom Side, Bottom-type and Valley-type Spread F, respectively, and that this classification should be kept in mind when comparing with results obtained using other instruments. For instance, Bottom-type and Valley-type (VHF radar) Spread F does not produce significant spread traces in ionograms or scintillations. They warned that considering these manifestations as a single phenomena responding to the same name, Spread F or FAIs, could lead to confusion. It was very possible that the physical mechanisms and instabilities responsible for the echoes could be different. The existence of different mechanisms 

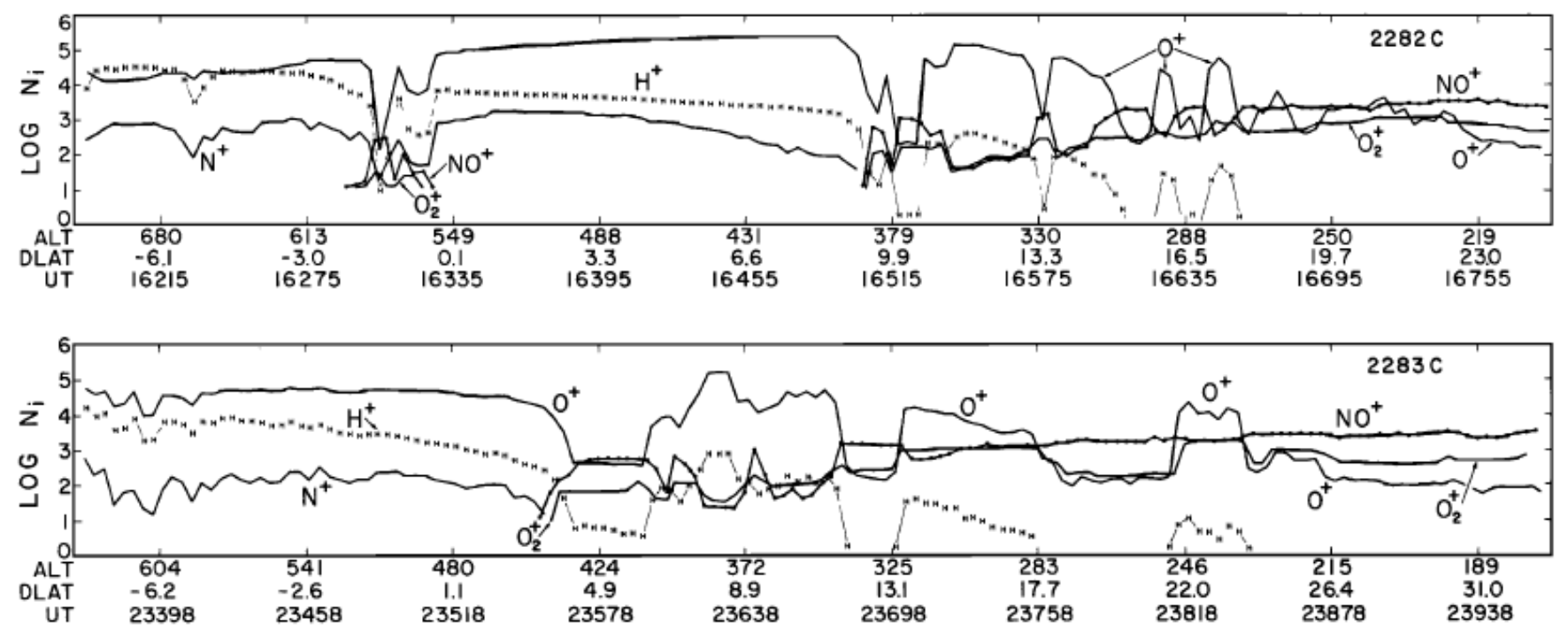

Fig. 9. Atmospheric Explorer $\mathrm{C}$ records of ionic composition showing very deep bite outs with a composition that is typical of the lower altitudes where they originated (from McClure et al., 1977).

was further supported by the large difference in the spectral signatures of the echoes. Spectral widths vary from a few Hertz to a few hundreds of Hertz (Woodman and La Hoz, 1976). It is difficult to accept that a single physical mechanism would be responsible for such a variety of manifestations, including their spectral width.

\section{Satellites, rockets, and other radars and radar tech- niques}

Considerable work has been done with in situ probes on satellites and rockets to study the nature of the irregularities responsible for Spread F. To do justice to these efforts would require a much longer review/tutorial than we have space for here. It suffices to mention that they have provided the only direct measurements of the intensity of the plasma density fluctuations and their one-dimensional scalesize spectrum. Previous to Woodman and $\mathrm{La} \mathrm{Hoz} \mathrm{(1976),} \mathrm{the}$ Atmospheric Explorer C (AE-C) team had measured depletions of electron density (Hanson and Sanatani, 1971, 1973; McClure et al., 1977) of close to three orders of magnitude at times. The depletions were large in size and had an ion composition that included molecular and heavy ions (Hanson and Sanatani, 1971) that corresponded to the composition of the lowest regions of the F-region. The low density and its composition pointed to a lower ionospheric origin. These measurements were crucial in the bubble interpretation given by Woodman and La Hoz (1976). Figure 9 taken from McClure et al. (1977) shows records of electron density showing very deep bite outs with an ionic composition that is typical of lower altitudes, revealing their origin. Although not showing well in these records, the bite outs usually show very steep boundaries, one side consistently steeper than the other.

The observation of asymmetrical slopes at a preferred side of a density fluctuation points to wave steepening as a mechanism in the production of smaller wavelengths (Costa and Kelley, 1978) and serves as a constraint in any theoretical model that would explain the cascading of primary instabilities to the small scales observed by VHF radars. Many onedimensional spatial plasma density spectra have been published, and many $k^{-n}$ like spectra with different densities and different slope break points have been found. Given the highly inhomogeneous nature of the fluctuations, I personally believe there is more information in the spatial series produced by the probes than in the k-spectra. Wave steepening and other manifestations of coherence between the different spatial spectral components are not evident in a simple spectrum (Costa and Kelley, 1978). One would require higher order cross spectra, which have not been calculated and would be difficult to interpret. One thing is clear, the observed plasma fluctuations are not similar to a Kolmogorov cascade type turbulence. Spectral shapes, wave steepening and their possible physical origin are extensively discussed in a review by Hysell (2000). There we also find a simulation showing how the intermediate wavelengths steepen (see Fig. 10).

Of particular importance to the question of long wave length gravity wave modulation of the bottom of the F-region (around $275 \mathrm{~km}$ altitude) prior to the development of bubbles and multiple-bubble patches, are the many multi-pass density and drift measurements made by the Atmospheric Explorer E (AE-E) (Singh et al., 1997). In Fig. 11 we show consecutive passes 7192 and 7193 . They show almost the same quasiperiodic density undulations with length scales 

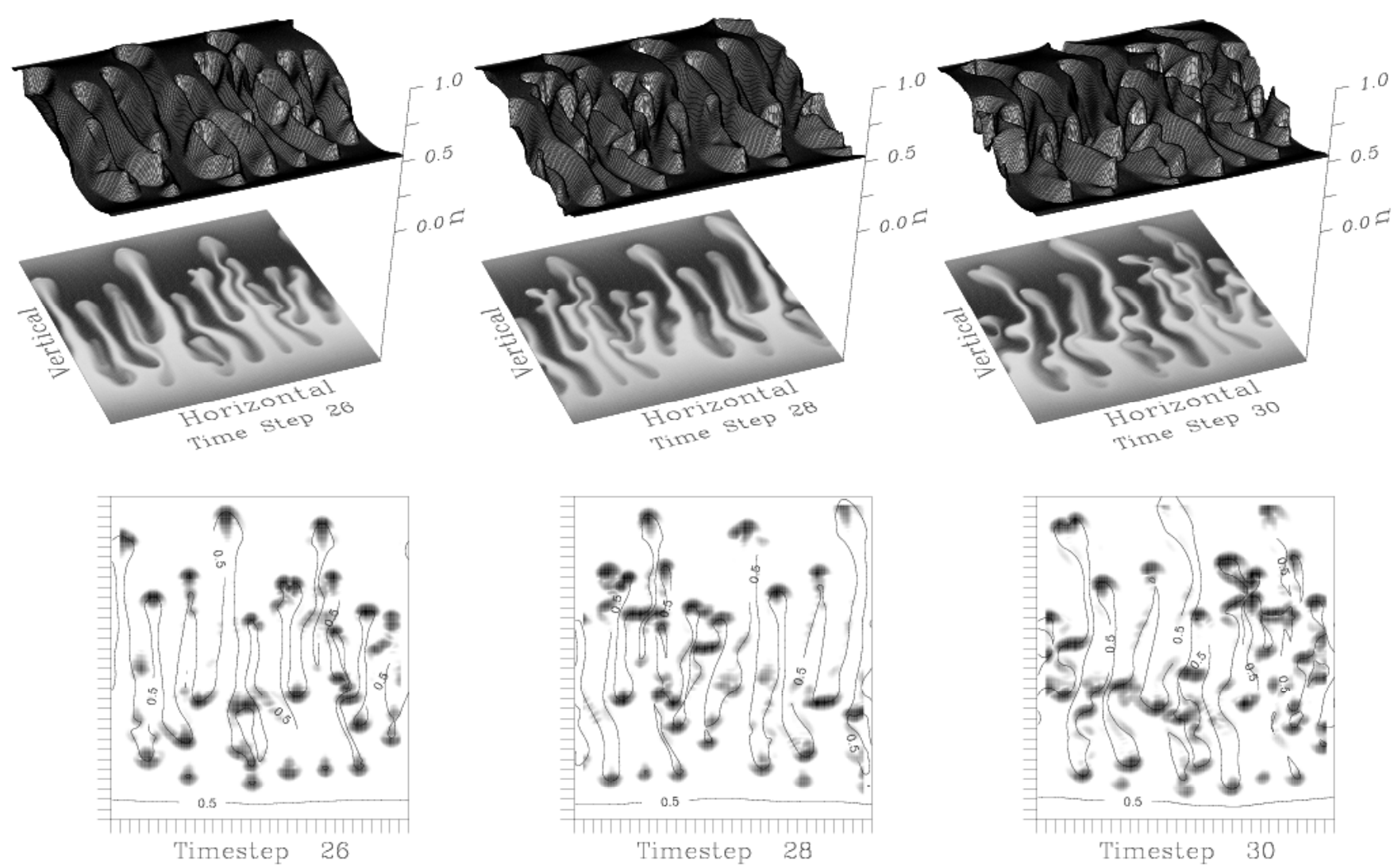

Fig. 10. Medium scale simulation of an interchange instability driven by gravity and a zonal electric field. Notice the teardrop formations with steep boundaries. They would be the location where meter-scale wavelengths would be formed (bottom panel) in discrete clusters. The size of the simulation is $6.28 \mathrm{~km}$ to a side (after Hysell, 2000).

from 150 to $800 \mathrm{~km}$, one around sunset and the next about 95 min later. The first is relatively free of smaller scale fluctuations, while the latter shows the development of bubbles or multiple bubble patches coincident with the original troughs in the large scale fluctuations. The periodicity and location of the large scale undulations is largely preserved.

AE-E can also measure neutral wind velocities. Figure 12 shows them for the same passes as in Fig. 11. Notice the same periodicities as compared with the large scale density fluctuations. These coincidences are used by Singh et al. (1997) to claim that gravity waves are the origin of the initial large scale wave fluctuations and that they provide the necessary seed for the development of the bubbles. This evidence should be kept in mind later on when we discuss current beliefs about the creation (without the need of gravity waves) of the initial seeding perturbations, considering that neutral wind perturbations cannot be produced by plasma instabilities. On the other hand, notice that there is no significant growth in the amplitude of the large scale electron density wave from one pass to the other and that the shorter scales are the ones that become unstable.

In the last two decades, the ALTAIR (US military) radar at Kwajalein has become available for limited scientific re- search (Tsunoda et al., 1979). This has become a very powerful tool for the study of the nature and evolution of Spread F. The radar operates at two frequencies, one at VHF $(155.5 \mathrm{MHz})$, and the other at UHF (415 MHz). Both can observe coherent echoes scattered back by the field aligned ionospheric irregularities when pointed perpendicular to the magnetic field, the former being more sensitive. But it is the capability to also obtain Incoherent Scatter electron densities, by looking off perpendicularity, that makes ALTAIR a unique and powerful tool (e.g. Tsunoda and White, 1981; Tsunoda, 2005). An additional advantage compared with Jicamarca RTI maps is its capability to cover, almost simultaneously, a two-dimensional crosscut of close to $1000 \mathrm{~km}$ in the EW direction at the bottom of the F-region and all altitudes of interest (e.g. Tsunoda, 1980; Hysell et al., 2005b, 2006a). One of their first contributions was to show that the enhanced back scatter was related to plasma bubbles, either using only radar data (Tsunoda, 1980) or with simultaneous plasma density satellite in situ measurements (Tsunoda et al., 1982) as shown in Fig. 13. In regards to the later, this correspondence had been shown previously using VHF radar and rocket measurements (Kelley et al., 1976; Morse et al., 1977), but only along a single one-dimensional cut as 

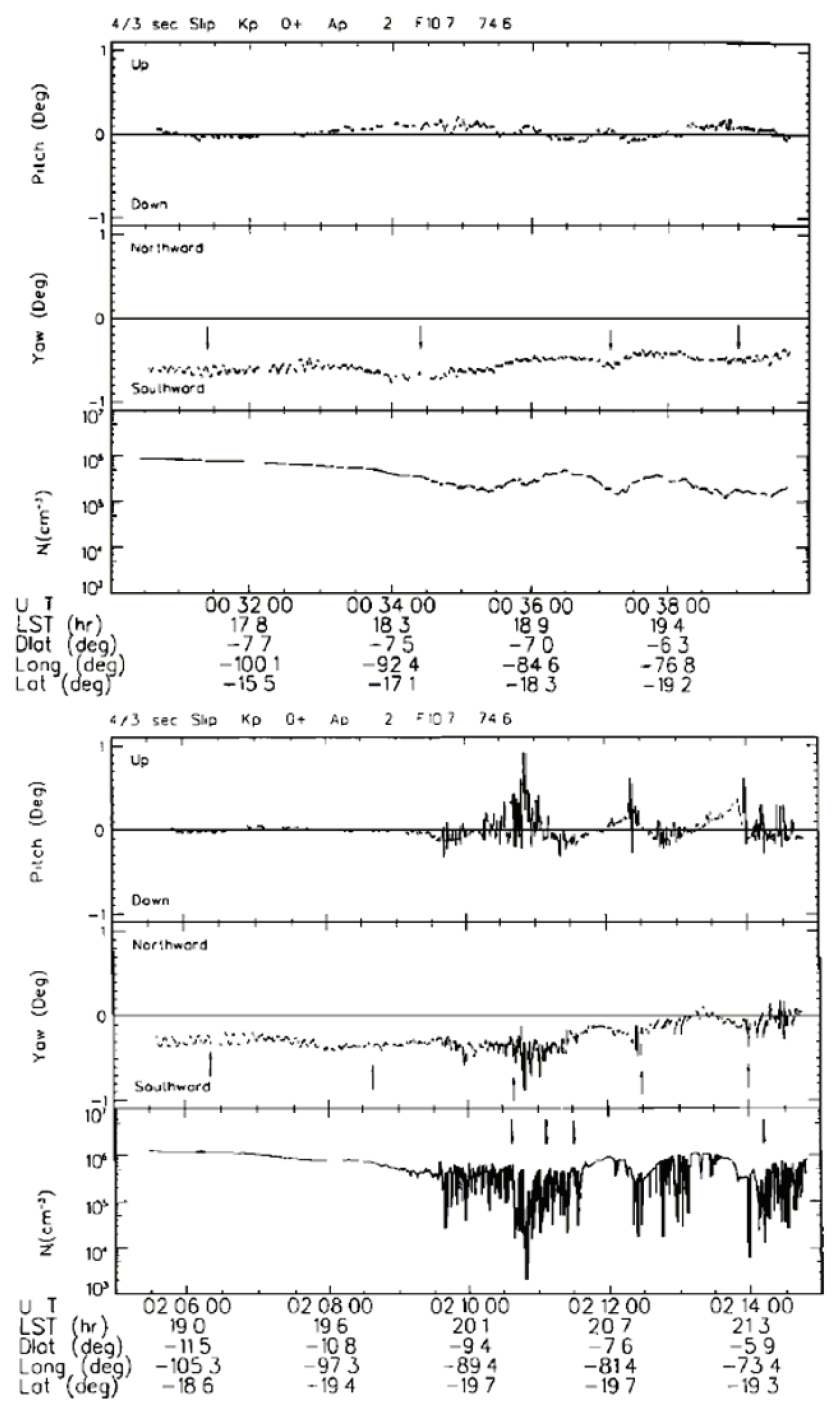

Fig. 11. Ion drift data and electron density plots taken during two consecutive orbits (7192 and 7193), almost retracing one another. The large scale fluctuations are very similar. The first orbit (top panel) shows undulations, attributed to gravity waves by the authors, that become unstable and break into large structured bubbles in the second orbit (after Singh et al., 1997).

compared to the 2-dimensional cuts provided by ALTAIR. Tsunoda (1980) showed evidence that the depletions were indeed narrow and elongated in the tilted vertical direction as postulated by Woodman and $\mathrm{LaHoz}$ (1976). The instrument is able to observe the background conditions when Spread $\mathrm{F}$ radar echoes start to appear. This is very important for understanding the day-to-day variability and the seeding responsible for the large scale wave growth under unstable conditions. The large scale waves have been shown to appear early in the evening before the Rayleigh Taylor instability has had much of a chance to make them grow. See for instance Fig. 14 from Hysell et al. (2005b). The existence of a

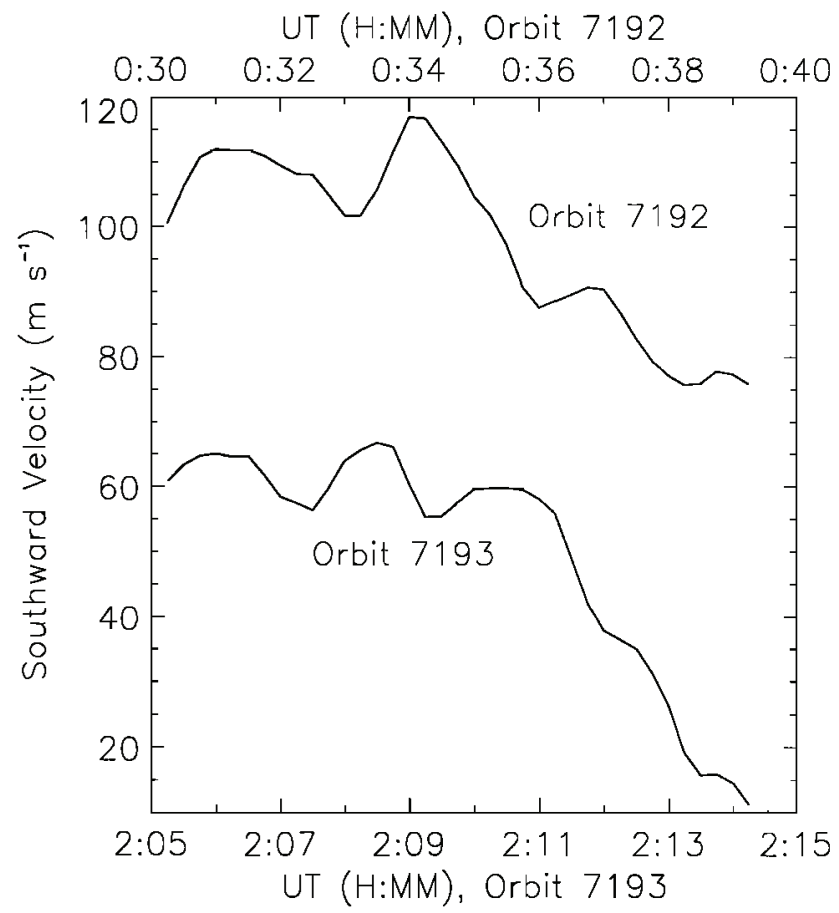

Fig. 12. Neutral winds measured by AE-E during the same two consecutive orbits shown in Fig. 10 showing similar undulations attributed to gravity waves (after Singh et al., 1997).

seed before Spread F break up is then an experimental fact as shown by this measurement and those of Singh et al. (1997), mentioned before.

Figure 14 also shows other interesting results. The figure shows a result from an experiment carried out at Kwajalein to study bottom type irregularities using ALTAIR and instrumented rockets (Hysell et al., 2005b, 2006a). An important feature that can be seen in Fig. 14 is the existence of weak bottom-type irregularities clustered into patches with the same periodicity as the one corresponding to the density waves above them. They have the same behavior as the ones observed at Jicamarca using imaging techniques (see below) whenever (and only when) bottomside Spread F occurs. They have been identified as a precursor and predictor of the later occurrence of Spread F, and hence their importance. The authors point out, additionally, that the bottom type layer does not occur at an altitude where the F-region bottomside gradient is the largest, but slightly below, where the westward ionospheric drifts are the strongest. This lack of coincidence can also be appreciated in the bottom type layers shown by Woodman and La Hoz (1976), when compared with the $h^{\prime}$ (almost the same as real $h$ at night time) as measured by an ionosonde, although the significance of the discrepancy was not realized then. It would appear then that a better name for the bottom-type irregularities should be "valley-top" instead. The electric field meter in the rocket showed a shear and a reversal of the zonal electric field and 


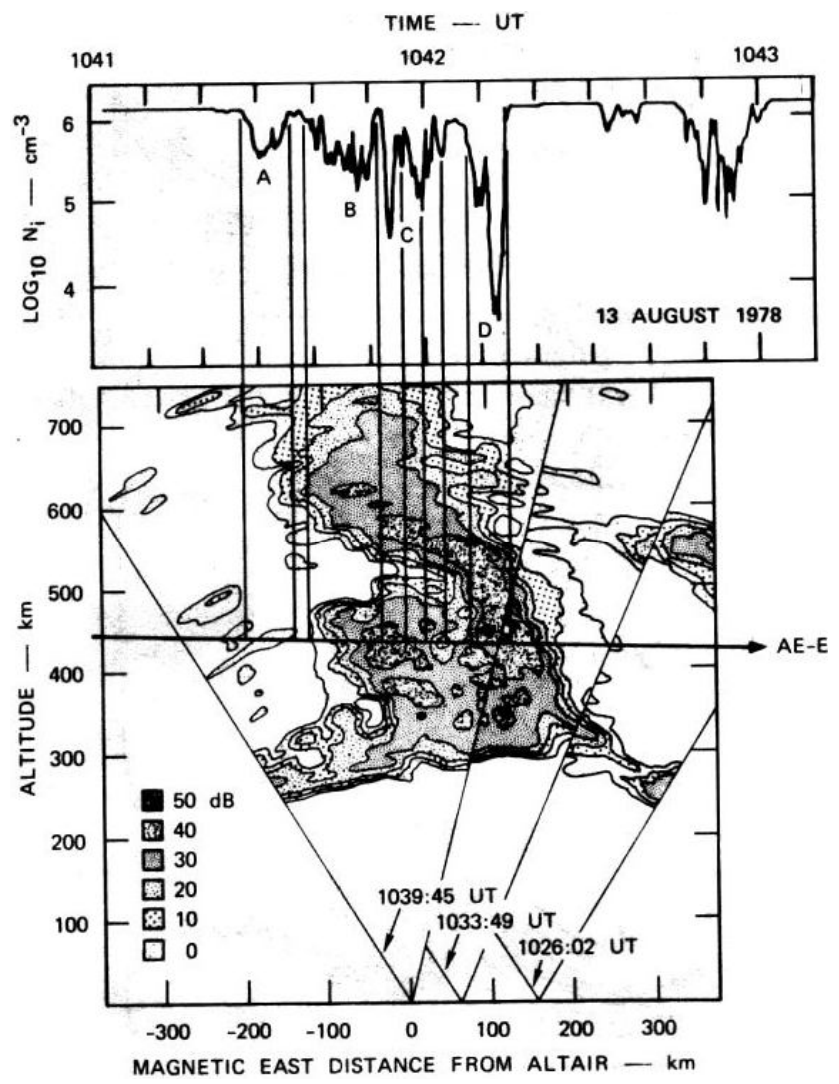

Fig. 13. The spatial relationship between Spread F depletions measured by satellites and backscatter power measured by the radar (after Tsunoda et al., 1982).

irregularities at the bottom of the F-region (see Fig. 15). The irregularities show an anisotropy in the short wave fluctuations in these irregularities, with the vertical electric field fluctuations being larger than the horizontal ones. These observations have a bearing on theories proposed recently for the formation of bottom type irregularities and the seeding of RT instabilities as discussed below.

In the last decade or so, two new VHF radars have been put into operation close to the magnetic equator, one by India, at Gadanki $\left(12.5^{\circ} \mathrm{N}\right.$ dip) and the other, the EAR radar, by the Japanese in Indonesia ( $23^{\circ} \mathrm{S}$ dip). They operate at 53 and $47 \mathrm{MHz}$, respectively. They are both phased arrays and can be steered electronically. This is a great advantage over Jicamarca.

The Gadanki radar is limited in their steering to positions in either of two planes, EW or NS. In the NS plane they can point perpendicular to the magnetic field at both E- and Fregion altitudes and obtain echoes from both regions simultaneously. By doing so they find that whenever Spread F bubbles appear, the E-region echoes weaken or disappear (Patra et al., 2004), as seen in Fig. 16. They explain the coupling in an indirect way as follows. The polarization field responsible for the uplift of the bubbles in its growth phase is not con- fined to F-region altitudes (e.g., Woodman and Chau, 2001), they involve lower altitudes in the valley region, including the apex of the flux tube that threads the E-region in the beam of the radar. The field propagates down to the E-region and stabilizes the instabilities there. This additional electric field must have an opposite polarity to the one responsible for the E-region irregularities. A similar experiment with similar results has been made with the EAR radar (Patra et al., 2005). There is still an open question on the reverse effect: how do the E-region irregularities at the foot of the flux tubes observed at the equator affect the formation of Spread F irregularities there? A correlation study was performed using the Piura VHF radar and Jicamarca simultaneously (Chau et al., 2002). Piura observed E- and F-region irregularities and Jicamarca the latter. No significant correlation was found, but the geometry was not the best; their magnetic longitudes are not close enough.

The EAR radar can steer to preselected positions from pulse to pulse. It does not have the limitations of the Gadanki radar and can point to an arbitrary azimuth. This permits the radar to follow perpendicularity to the field lines east and west of the magnetic meridional plane (Fukao et al., 2003; Fukao et al., 2004). Although their steering angles are not as large as those obtained with the ALTAIR radar, the radar can also produce what is effectively a time sequence of snapshots of the irregularities. This is an advantage over the Jicamarca radar. It should be remembered that this is not what the RTI plots from Jicamarca show. It would be the case if the form of the irregularity patches was frozen to an East-West plane that is drifting by Jicamarca at a constant and uniform speed. This is a rough approximation that helps to interpret the RTI plots, but it can deviate considerably from reality, especially when we look at large scale features in the EW dimension. A patch of irregularities can be seen when it transients by a fixed beam like Jicamarca's, but we cannot tell when and where it was created. In contrast, with an antenna that can also see East, West, and vertical, one can see FAI formation before it shifts eastward to the vertical beam and its further development to the East. The EAR radar has made use of this potential and has determined that plumes start to form very close to the E-region terminator (Yokoyama et al., 2004).

Jicamarca's RTI plots have been interpreted as slit-camera pictures of the irregularities with the limitations mentioned above. They are distorted not just because different patches move at different velocities, but also because they appear and disappear during the lapse time of the RTI. This limitation - without underestimating its proven usefulness - has been removed with a recent development that effectively converts the radar into a radar camera (Hysell, 1996; Woodman, 1997), albeit with a reduced angular coverage. The images obtained cover an angle not wider than that of an antenna module $(1 / 8 \times 1 / 8$ of the full area) or, as has been the case so far, by the beam pattern of the transmitter antenna (about $6^{\circ}$ ), configured especially for this purpose. On reception, the technique uses only a few modules with different 

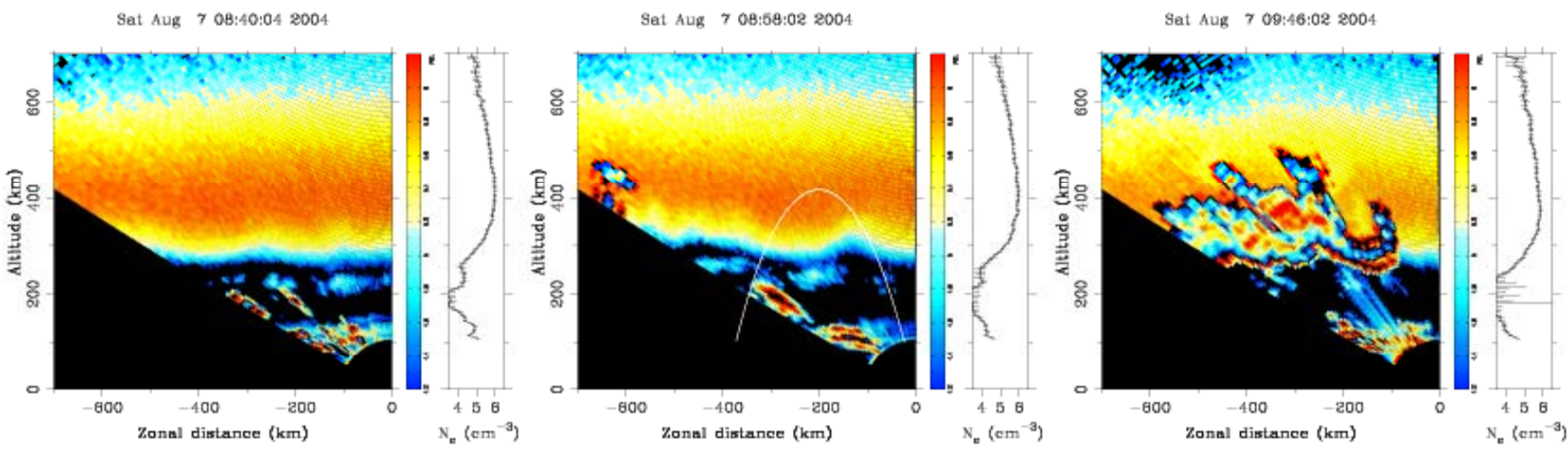

Fig. 14. ALTAIR consecutive scans made before, during and after an instrumented rocket flown on 15 August 2004, showing the existence of an undulation of the bottom side of the F-region, coincident with bottom-type Spread F patches, and the later development of plumes (after Hysell et al., 2005b).
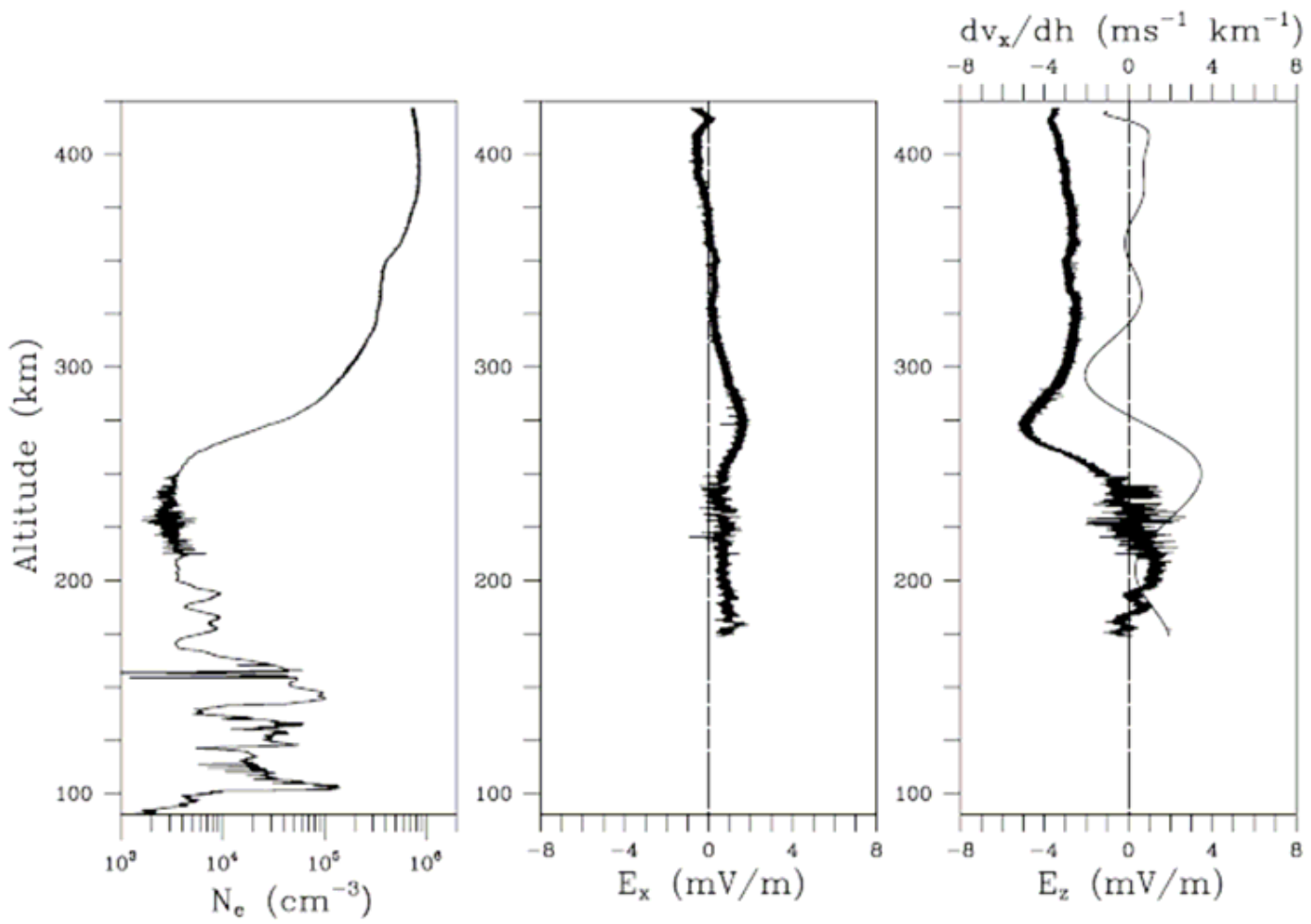

Fig. 15. Rocket measurements of electron density and electric field coincident with the radar scan of Fig. 13. Notice the location of the bottom-type irregularities coincident with the altitudes having a westward drift. Notice also the anisotropic electric fields (and drifts) (after Hysell et al., 2005b).

EW projected positions and different separations in between. A one-dimensional (EW) spatial correlation function of the diffraction pattern is obtained at different spatial "lags". Its spatial Fourier transform is then estimated (Inversion techniques are necessary since not all desirable lags are measured) to get the brightness image one wishes to obtain.

The resolution of the image is determined by the largest separation between the modules, and with the use of the
Maximum Entropy technique, one obtains a resolution close to an order of magnitude better than the beamwidth of the full antenna. The resolution has been improved by a factor of two with the construction of an additional antenna with the same design as a module, lengthening the previous largest baseline by $200 \mathrm{~m}$ (Hysell and Woodman, 1997).

Breaking our chronological approach we reproduce in Fig. 17 one of the latest images of Spread F obtained with this 

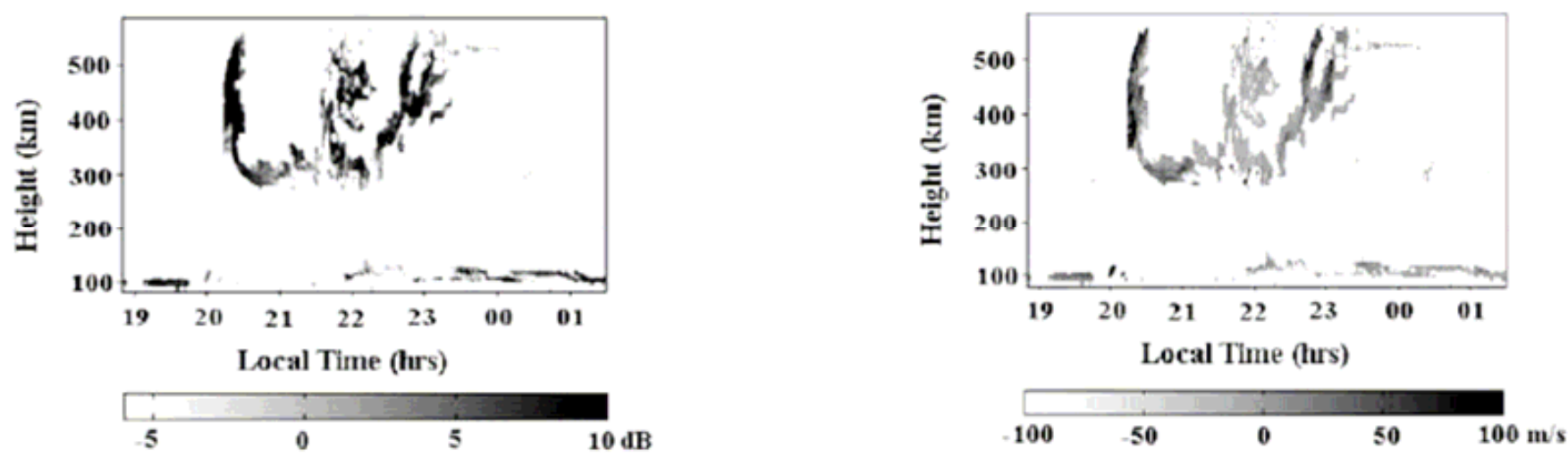

Fig. 16. Power and drifts of F- and E-region backscattering echoes from FAI. Notice the supression of the E-region echoes when the velocity of the F-region is downwards (after Patra et al., 2004).
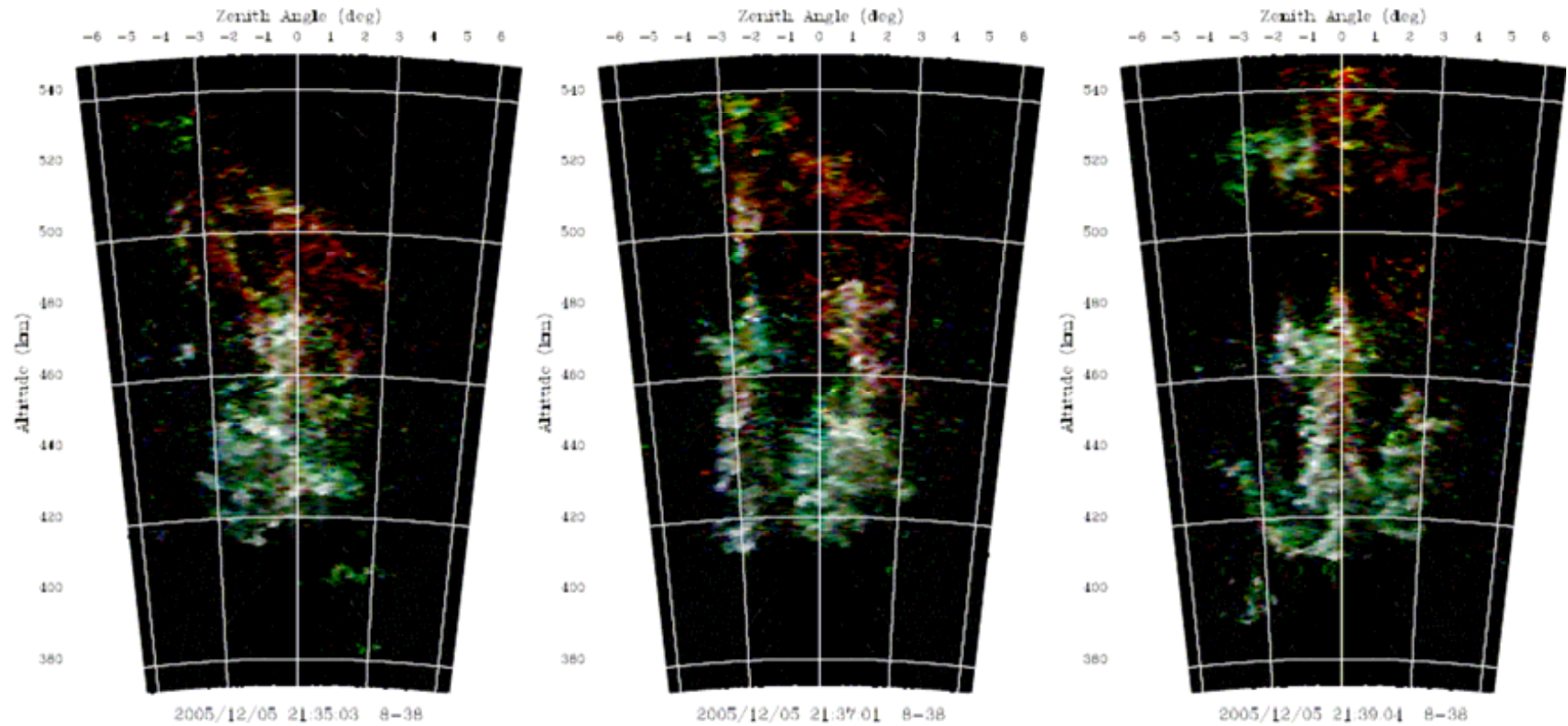

Fig. 17. Jicamarca interferometric image showing a Spread F plume. Notice the plume bifurcation and the rapid uplift of the red (negative Doppler shift) patch from one frame to the next (courtesy of D. L. Hysell).

technique, including the latest refinements (Hysell and Chau, 2006). Note that the system produces a sequence of images at consecutive times, which can be played as a movie. By spectrally analyzing the signals in time, one can obtain different images for different Doppler shifts. By assigning different colors to the different Doppler shifts, one can obtain a composite picture which carries information about the radial velocities associated with the different pixels of the image.

Before continuing with the contributions made with the imaging technique, it should be mentioned that despite the limitations of the Jicamarca radar, when used in the slit camera mode, it has made recently an important contribution to the understanding of the initiation of Spread $F$ as a result of an improved technique to measure vertical and EW drifts (Kudeki et al., 1999). Figure 18 taken from Kudeki and Bhat- tacharyya (1999), is an example of the new results, where the drift velocity field, made with improved accuracy and altitude resolution, is superimposed on the electron density background measured using incoherent scatter techniques. Coherent echoes produced by Spread F irregularities are also shown using a different color palette. The vortex shown at the bottom of the F-region at the initiation of bottom type Spread F is typical of many nights reported in the reference. The paper has initiated a series of others stressing the importance of the vertical shear in the EW ionospheric drifts in the bottom of the F-region. Most important is the clear evidence of the existence of westward drifts in an eastward moving neutral background and its relationship to the initiation of Spread F irregularities. 
The current capability of Jicamarca to obtain real images with the best zonal resolution of all the radars mentioned above has been exploited by Hysell and colleagues (Hysell, 1996, 1998, 1999; Hysell and Woodman, 1997) to study the structure or the lack of structure within radar plumes or other type of Spread F patches. These images have shown, for instance, the existence of high velocity plasma drifting upwards in the central region of a plume and much smaller velocities on the walls. Other features that are evident in the images are the bifurcation of plumes and the pinching of very narrow channels at the neck of the plume where supposedly low density plasma is feeding the head of the plume. The good zonal resolution also shows that bottom type irregularities, those that do not develop later into bottomside Spread F, are fairly homogeneous in the zonal direction, showing anisotropic structures elongated in the EW direction and drifting to the west. In contrast, when they do develop into bottomside irregularities or plume-like structures, they break up into patches of a few tens or more kilometers in the zonal direction (Hysell et al., 2004). The scale sizes of the patches are equivalent to those of the plumes, indicating that they are modulated by the same undulation that seeded the plumes. This conforms to the observations made in Kwajalein and mentioned before. The existence of the patchy structures as a precursor to the development of bottomside Spread F and plumes could be used as a predictor for the latter.

In addition to the above techniques, the operation of the Jicamarca radar in JULIA mode has made an enormous contribution by documenting hundreds of night time records of Spread F as seen by the radar. JULIA, conceived and implemented by B. B. Balsley, uses the large Jicamarca antenna with a low power transmitter, unattended. Lately, it does so in an interferometer mode. An example of its potential can be appreciated in Hysell and Burcham (1998). The variety of RTI shapes presents a challenge, which we wish one day to be able to fully understand. The data are available on the Web (http://jro.igp.gob.pe/julia).

Propagation studies of satellite signals at different frequencies allow the measurement of the total electron content (TEC), integrated along the path from the satellite to a receiver site. The technique is sensitive to the existence of electron density bubbles along the path, which produce a significant depletion in the measured TEC. Networks of receivers deployed at different equatorial latitudes and at adjacent longitudes can be used to measure the location, extension and intensity of such depletions. Valladares et al. (2004) has used a network of GPS receivers around the equatorial $75^{\circ} \mathrm{W}$ meridian to study the occurrence of satellite scintillations and TEC depletions in the context of different developments of the equatorial anomaly. Using JULIA, the authors show a close correspondence between radar plumes, TEC depletions and the occurrence of scintillations. The background TEC is used as a proxy of the development of the equatorial anomaly and its day to day variations. Qualitative case stud-

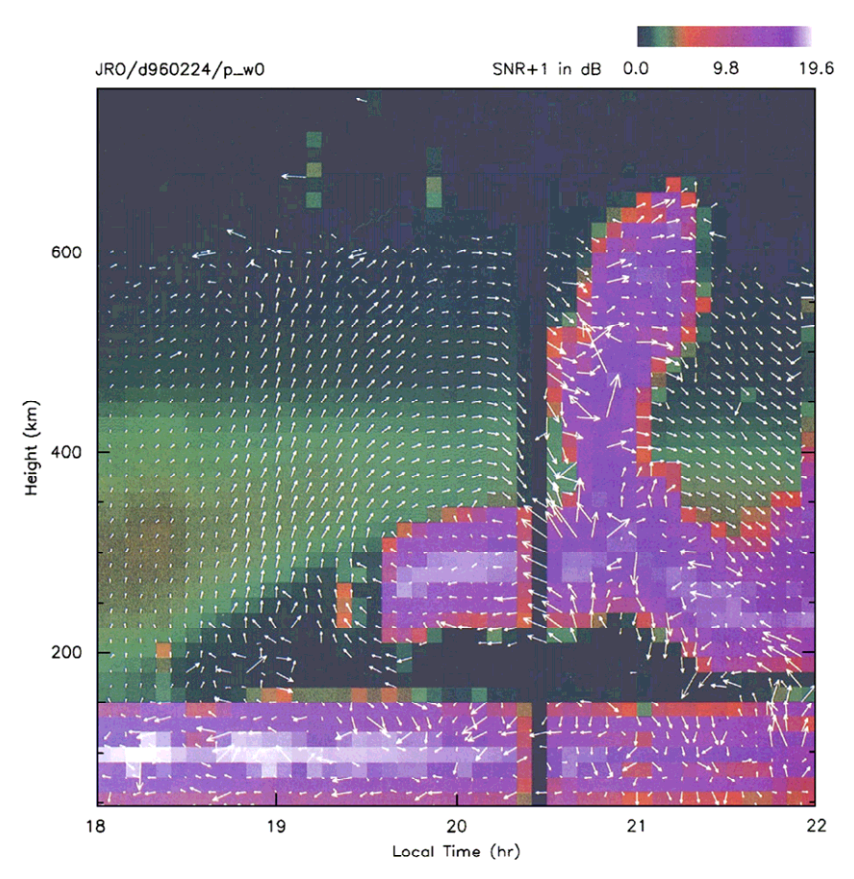

Fig. 18. RTI plot showing Spread F echoes (purple color), electron density (greenish tones) and vector drifts. Note the very clean vector drifts in the F-region. The main features of this image is the existence of a vortex at the bottom of the F-region, with westward drifts in the lower side, right before the initiation of bottom-type Spread F echoes (after Kudeki and Bhattacharyya, 1999).

ies show the importance of Hearendel's (1973) magnetic flux tube integrated densities in determining the stability of the F-region.

All-sky airglow optical images taken of the $6300 \AA$ ionospheric emission provide a powerful technique to obtain twodimensional images of the depleted regions of the ionosphere associated with the radar plumes and Spread F. Depletions show as dark bands or regions in the all-sky images. By assuming that the emissions come from a relatively narrow layer around $300 \mathrm{~km}$ and that the depletions are elongated along the magnetic field line, it is possible to map the horizontal images to an image of the depletions in the (magnetic) equatorial EW-vertical plane. The technique complements nicely the radar images, not only showing the large scale morphology of the depletions but the assumed correspondence between these depletions and the small scale irregularities responsible for the radar echoes (see for instance Mendillo et al., 2005; Makela, 2006, and references therein).

\section{Recent theoretical efforts}

Theoretical work related to Spread $\mathrm{F}$ in later years has been concentrated in two periods, one right after the plume observations of Woodman and LaHoz (1976), and the other in the last 5 to 10 years. The first period includes mainly the efforts 

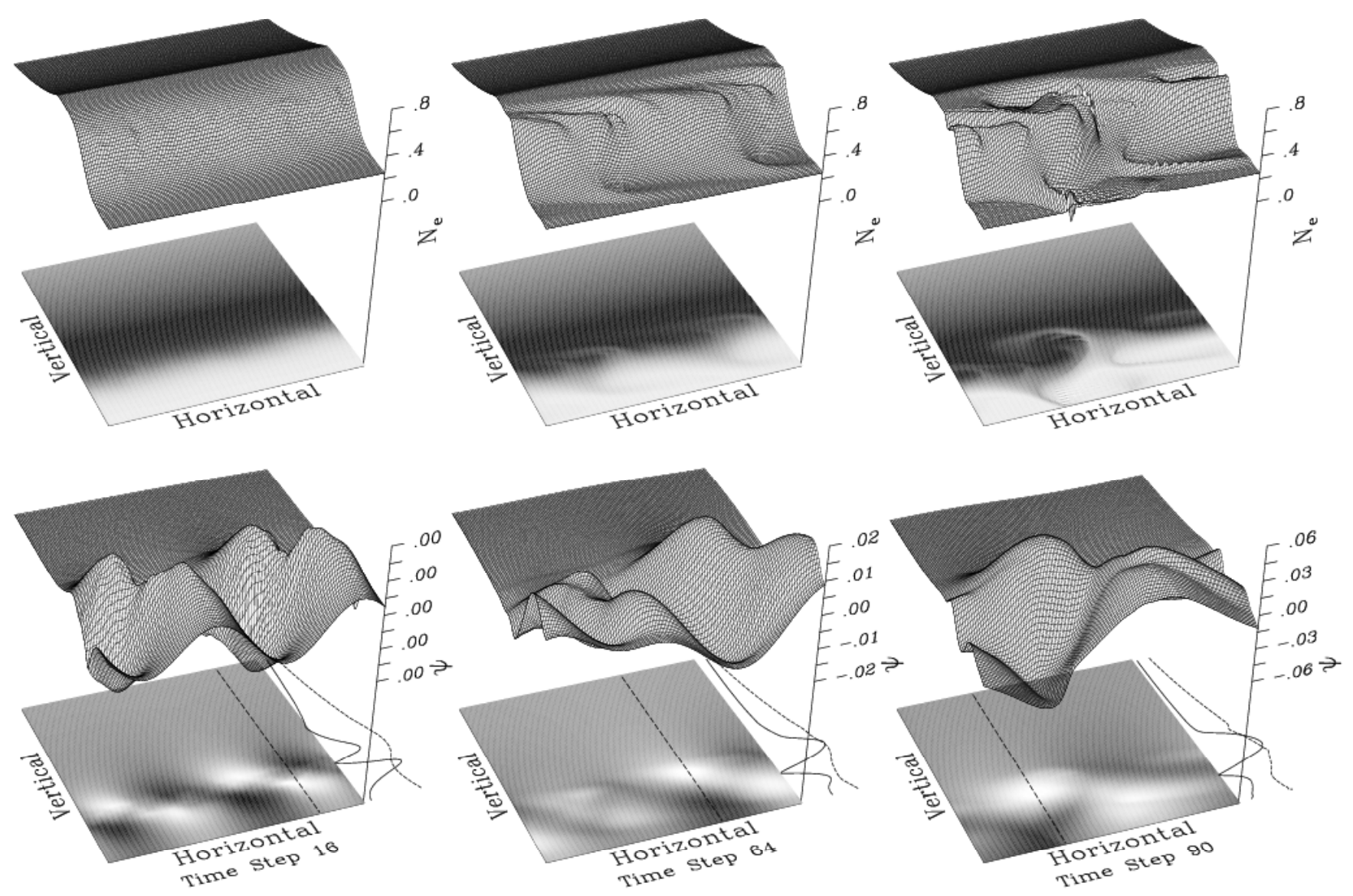

Fig. 19. Numerical simulation showing the development of a shear collisional instability in the bottom of the F-region. The model includes a sheared zonal plasma drift profile reversing to westward at lower altitudes, an altitude varying collision frequency and an eastward neutral wind. The top panel represents the plasma density, and the bottom, a stream function, which controls the drift velocity perturbations. Notice the $45^{\circ}$ quasi-sinusoidal waves changing from a transient behavior to longer wavelength during the steady state. Transient and steady states have been identified before in linear analytical mathematical models involving shear flows (after Hysell and Kudeki, 2004).

of the NRL group mentioned earlier (see Ossakow, 1981, for a review) to model numerically the formation of bubbles with increasing degrees of sophistication. It was found that the growth rate of the RT instability or even the GRT instability was too slow to explain the degree of development just about an hour after sunset at E-region heights (Sultan, 1996). The existence of large amplitude undulations in electron density produced by gravity waves was postulated as a seed from which the instability would grow (e.g. Kelley et al., 1981) and many efforts were made to find evidence that such was the case. Variations in gravity wave activity was postulated as an explanation for the variation in Spread F occurrence even under otherwise similar measured background conditions, a role that is difficult to prove, considering our inability to measure directly gravity wave presence at the altitudes where Spread F is initiated.

The second, more recent, theoretical period was triggered by Kudeki and Bhattacharyya (1999), with their vortex pictures (e.g., Fig. 18) and the realization that the counter streaming of westward drifts and eastward neutral winds, at the bottom and valley of the F-region, was another important energy source for creating instabilities.

The current understanding comes mainly from the efforts of Hysell, Kudeki and colleagues (Kudeki and Bhattacharyya, 1999; Hysell and Kudeki, 2004; Hysell et al., 2005a, b, 2000a, 2006b; Kudeki et al., 2007). Hysell and Kudeki (2004) identified a collisional shear instability at the shear region of the F-region that could compete with the GRT in this region and performed numerical simulations to illustrate their growth (see Fig. 19), although the values obtained were not very impressive. A surprising result was the appearance of quasi sinusoidal waves tilted by $45^{\circ}$.

Recently Hysell et al. (2006b) obtained an approximate analytical solution to the same non-local problem using realistic values for the background condition and found that $45^{\circ}$ waves would be most unstable, with growth times that would be of the order of $6 \mathrm{~min}$ for the longer wavelengths $(\sim 200 \mathrm{~km})$ and maximizing at altitudes $30 \mathrm{~km}$ lower than the 


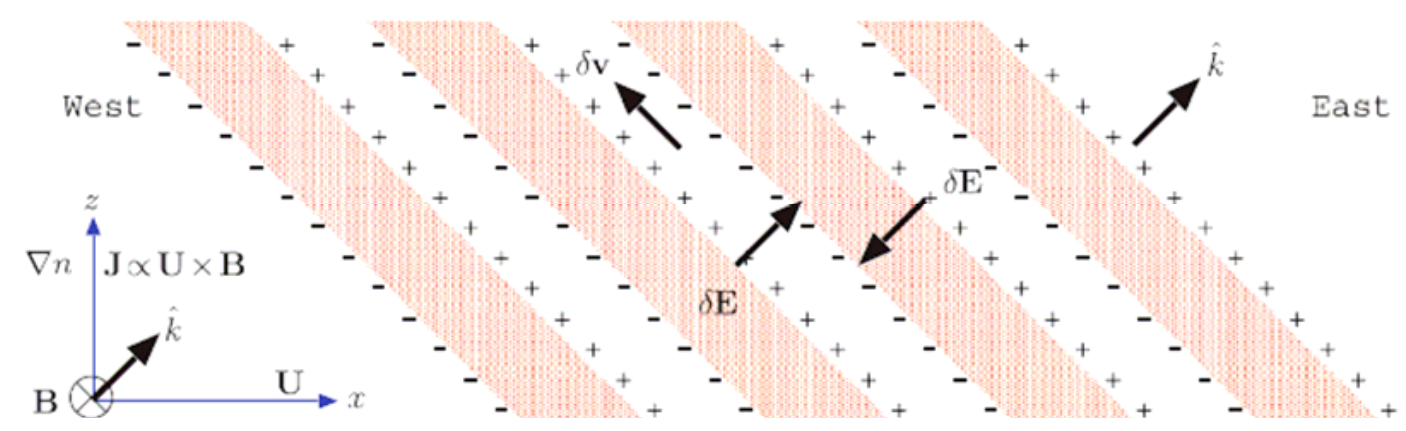

Fig. 20. Cartoon showing the physical processes responsible for the "collisional shear instability" which has been proposed to seed a later GRT instability. The mechanism is as follows: The wind, $U$, produces a vertical current, $J$, the current polarizes the wave fronts, the polarization field, $\delta E$, produces drifts, $\delta v$, along the fronts, increasing the original amplitude of the wave perturbation, increasing, in turn, its polarization and consequently the amplitude of the wave perturbation (after Kudeki et al., 2007).

GRT instability. They propose that this instability would undulate the bottom of the F-region and seed later a GRT growth. For the background conditions, Hysell et al. (2006b), has developed a 3-D model of the evening equatorial ionosphere, which is by itself an important contribution to equatorial F-region dynamics. They adjusted the parameters to conform with the measured values obtained by the Kwajalein IS and rocket experiments.

More recently Kudeki et al. (2007) obtained comparable growth times using a linear non-local approach to the problem including altitude dependent gradients, collision frequencies and sheared plasma drifts, and emphasized that it is the counter streaming rather than the shear that is mainly responsible for the instability. It is effectively a wind driven interchange instability, but this alternative name is already being used to describe the instability responsible for the bottom-type Spread F discussed below. It is in this regard that the Kudeki et al. (2007) emphasis becomes important since the name, collisional shear instability, is confusing.

The physical process responsible for the instability can be understood by means of the cartoon shown in Fig. 20 taken from Kudeki et al. (2007). The background conditions are given by a vertical plasma density gradient and a neutral wind blowing horizontally and perpendicular to the gradient and the magnetic field. They represent the gradient at the bottom of the F-region and the relative eastward neutral wind blowing on a westward moving plasma as has been observed at Jicamarca and Kwajalein. The condition is unstable. Waves with phase fronts tilted $45^{\circ}$ to the west have the fastest growth. The wind produces a vertical current, which polarizes the wave fronts. The polarization field produces drifts along the fronts, increasing the original amplitude of the wave perturbation, increasing in turn its polarization and consequently the amplitude of the wave perturbation. For a gradient scale lengths of $20 \mathrm{~km}$ and a $200 \mathrm{~m} / \mathrm{s}$ wind, Kudeki et al. (2007) estimate a growth characteristic time of $200 \mathrm{~s}$, which is equivalent to a growth of $18 \mathrm{e}$-folds in an hour. The more realistic linear and non local mathemat-

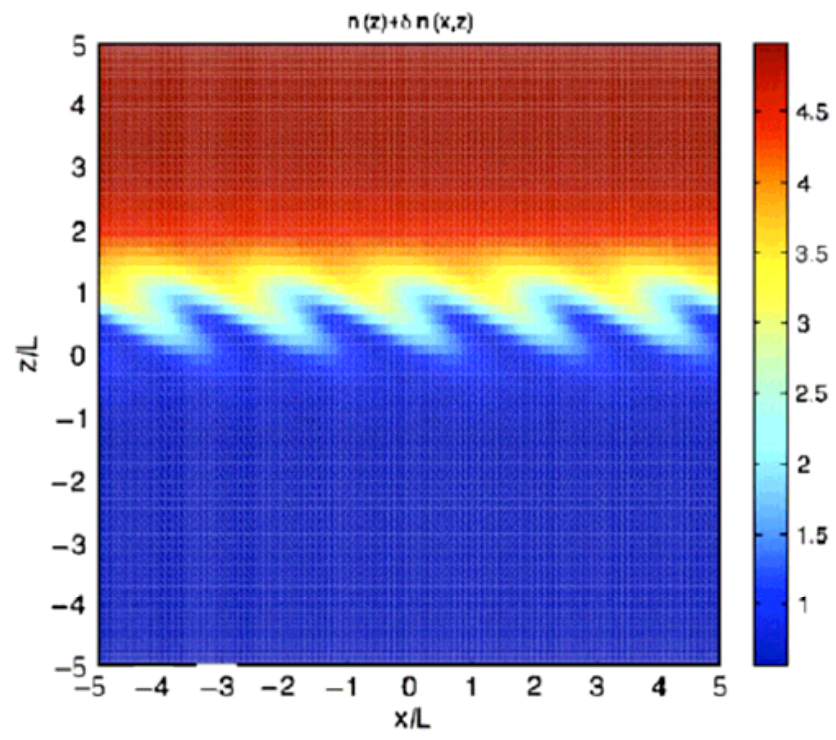

Fig. 21. Plasma density fluctuations resultant from a non-local linear perturbation model of the "collisional shear instability". Dimensions are normalized with respect to the gradient scale length, $L$. For $L=20 \mathrm{~km}$, a neutral wind of $200 \mathrm{~m} / \mathrm{s}$, vertical drift of $20 \mathrm{~m} / \mathrm{s}$, gravity included, the growth rate is equivalent to $18 \mathrm{e}$-folds in an hour. No zonal plasma drift (and no shear) is included (after Kudeki et al., 2007).

ical model, which includes an altitude dependent collision frequency and a neutral velocity shear decreases the growth rate (shear stabilizes the process), but comparable values are recovered when gravity and a typical zonal electric field is included. Figure 21 shows the plasma density solution under this latter condition, for a wavelength equal to twice the gradient scale length, $40 \mathrm{~km}$. The $45^{\circ}$ tilted quasi sinusoidal wave of the simplified model is still there. For longer wavelengths, of the order of $200 \mathrm{~km}$, Kudeki et al. (2007) estimate growth times of the order of a few minutes. These authors also postulate that this instability provides the seed to the 
GRT instability for the later growth and non linear development and that there is no need of a gravity wave to provide the seeding, other than to increase the level of the geophysical noise above the thermal level, shortening the developing time even more.

The bottom-type Spread F is also produced by the counter streaming, in this case as a consequence of a wind driven interchange instability produced by the differential neutral wind working on horizontal gradients (Kudeki and Bhattacharyya, 1999). The unstable waves are vertical and appear as horizontal striations, just as observed by the Jicamarca radar in the imaging mode. These irregularities show patchiness on nights that bottomside Spread F or plumes develop. The idea is that they are modulated by the same unstable long and medium wavelengths mentioned above that seed their later RT unstable response.

\section{Theories for the small wavelength scales}

Most of the theoretical literature about Spread F is concerned with the formation of large and medium scale irregularities, their morphology and possible physical mechanisms that produce them, but little has been done to explain the formation of the irregularities responsible for the radar echoes ( $3 \mathrm{~m}$ for Jicamarca and as small as 35 and $11 \mathrm{~cm}$ for the radars at Kwajalein) and yet we use them as a diagnostic of what is happening at the larger scales. We implicitly assume that there is a cascade mechanism as proposed by Haerendel (1973) from the larger to the smaller scale, but we do not know exactly how this takes place. From numerical simulations and in situ measurements we know that the irregularities steepen. See for instance the simulation by Hysell (2000), shown in Fig. 10. But numerical simulations use fluid equations which we know are not valid for wavelengths comparable to or smaller than the ion gyro radius (about $6 \mathrm{~m}$ ), and the in situ measurements are not able to resolve scale lengths this small. Drift waves have been postulated to explain a break down from the steep gradients of the larger scales (Costa and Kelley, 1978a), and a kinetic approach (Costa and Kelley, 1978b) has been taken to prove their existence. Huba and Ossakow $(1979,1981)$ have considered different hybrid modes including collisions, opening the possibility for a certain range of wave numbers and excluding others, which had been considered before. The current state of the theory is that high frequency drift instabilities can explain the shortest wavelengths, up to $\sim 1 \mathrm{~m}$ and the low frequency waves longer than $10 \mathrm{~m}$, but no existing theory can explain the waves around $3 \mathrm{~m}$, i.e., the strong echoes that Jicamarca sees! (Ossakow, 1981, and references therein). There is also a constraint that the high frequency waves have to meet, the very narrow frequency spectra of the bottomtype and some bottomside radar echoes, with Doppler offsets that do not differ much from those of the background.

\section{Where do we stand?}

The progress has been very slow. Seventy years have gone by since the discovery of Spread F irregularities, but we are getting there. We have a good understanding of the formation of bottom side irregularities and plumes through numerical simulations of GRT instabilities. But we would like to understand better the day to day variability. A good theory in physics should be able to make good predictions, and we are not in that position yet. Until recently we have been putting the burden on a variable existence of gravity waves to do the seeding. Now we know that the neutral wind at the bottom of the F-region can do that job. In fact neutral winds have become triply important (Kudeki et al., 2007). They are responsible for the pre-reversal enhancement of the vertical velocities and consequent uplift of the F-layer to more unstable altitudes, for the westward reversal of the ionospheric drift at the bottom of the F-region and as a source of instabilities that seed the later GRT development. We have also learned that the neutral wind is responsible for the bottom-type and valley-type irregularities working on any zonal gradient of the electron density (Kudeki and Bhattacharyya, 1999; Hysell et al., 2006a).

Unfortunately neutral winds have been difficult to measure in the past, but fortunately, night airglow measurements should soon be able to do the job. A modern airglow instrument (SOFDI, J. Meriwether, personal communication) is going to be deployed near Jicamarca and it should be able to correlate changes in neutral wind velocity with the three effects mentioned above. In addition, C/NOFS has recently been launched with special instrumentation to study spread $F$, including neutral winds. Furthermore, the construction of a "distributed observatory," composed of GPS receivers, ionosondes and magnetometers displayed along the $75^{\circ} \mathrm{W}$ meridian and neighboring sites (project LISN, Valladares and colleagues, personal communication) should allow its investigators to measure or infer the state of the F- and E-region at the foot of the field tubes that go unstable. Of particular importance is the determination of the integrated density along the magnetic field lines and the stabilizing conductivities at their ends. We should then, in the near future, knowing the state of the ionosphere and the neutral atmosphere, be able to predict when Spread F is going to occur or not, or at least compare, retroactively, model simulations of spread $F$ with its actual behaviour. In regards to the very small length scales responsible for the radar scattering, additional research is needed to conform theoretical expectations with what is being observed.

Acknowledgements. The author thanks David Hysell and Erhan Kudeki for elucidating discussions about the recent theories of F-region instabilities, and David Hysell, Jorge Chau, and Don Farley for their critical reading of earlier versions of the manuscript. The Jicamarca Radio Observatory is a facility of the Geophysical Institute of Peru, Ministry of the Environment, and it is operated with support from the NSF Cooperative Agreement ATM-0432565 
through Cornell University.

Topical Editor K. Kauristie thanks two anonymous referees for their help in evaluating this paper.

\section{References}

Aarons, J.: Equatorial scintillations: A review, Antennas and Propagation, 25, 729-736, 1977.

Abdu, M. A., Reddy, B. M., Walker, G. O., Hanbana, R., Sobral, J. H., Fejer, B. G., Woodman, R. F., Schunk, R. W., and Szuszcewicz, E. P.: Processes in the quiet and disturbed equatorial-low latitude ionosphere: SUNDIAL, campaign 1984, Ann. Geophys., 6, 69-80, 1980.

Basu, S. and Basu, S.: Equatorial Scintillation-a review, J. Atmos. Terr. Phys., 43, 473-489, 1981.

Basu, S. and Basu, S.: Equatorial Scintillation: advances since ISEA-6, J. Atmos. Terr. Phys., 47, 753-768, 1985.

Booker, H. G. and Wells, H. W.: Scattering of radio waves by the F region of the ionosphere, J. Geophys. Res., 43, 249-256, 1938.

Booker, H. G.: Turbulence in the ionosphere with applications to meteor-trails, radio-star scintillation, auroral radar echoes and other phenomena, J. Geophys. Res., 61, 673-705, 1956.

Booker, H. G.: The use of radio star to study irregular refraction of radio waves in the ionosphere, Proceedings of the IRE, 46, 298-314, 1958.

Bowles, K. L., Ochs, G. R., and Greeen, J. L.: On the absolute intensity of incoherent scatter echoes from the ionosphere, Journal of Research of the National Bureau of Standards - Radio Propagation, 66D, 395-407, 1962.

Cohen, R. and Bowles, K. L.: Ionospheric VHF scattering near the magnetic equator during the International Geophysical Year, Journal of Research of the National Bureau of Standards, 67D, 459-480, 1963.

Costa, E. and Kelley, M. C.: On the role of steepened structures and drift waves in equatorial spread F., J. Geophys. Res., 83, 43594364, 1978a.

Costa, E. and Kelley, M. C.: Linear theory for collision less drift waves with wavelengths near the ion gyroradius, J. Geophys. Res., 83, 4365-4368, 1978b.

Chaturvedi, P. K. and Ossakow, S. L.: Nonlinear theory of the collisional Rayleigh-Taylor instability in equatorial spread-F, Geophys. Res. Lett., 4, 558-560, 1977.

Chau, J. L., Woodman, R. F., and Flores, L. A.: Statistical characteristics of low-latitude ionospheric field-aligned irregularities obtained with the Piura VHF radar, Ann. Geophys., 20, 12031212, 2002, http://www.ann-geophys.net/20/1203/2002/.

Dungey, J. W.: Convective diffusion in the equatorial F region, J. Atmos. Terr. Phys., 9, 304-310, 1956.

Farley, D. T., Balsley, B. B., Woodman, R. F., and McClure J. P.: Equatorial spread F: Implications of VHF radar observations, J. Geophys. Res., 75, 7199-7216, 1970.

Freemouw, E. J. and Rino, C. L.: Development of a worldwide model for F-layer produced scintillation, final report, Stanford Research Institute, Menlo Park, Calif., N-72-23373, 128 pp., 1970.

Fukao, S., Ozawa, Y., Yamamoto, M., and Tsunoda, R. T.: Altitude extended equatorial. Spread F observed near sunrise terminator over Indonesia, Geophys. Res. Lett., 30, 2137, doi:10.1029/2003GL018383, 2003.
Fukao, S., Ozawa, Y., Yokoyama, T., Yamamoto, M., and Tsunoda, R. T.: First observations of spatial structure of $F$ region 3-m-scale field-aligned irregularities with the Equatorial Atmosphere Radar in Indonesia, J. Geophys. Res., 109, A02304, doi:10.1029/2003JA010096, 2004.

Haerendel, G.: Theory of equatorial spread F, Max-Planck Institute für Physik und Astrophysik. Garching, West Germany, preprint, 1973.

Hanson, W. B. and Sanatani, S.: Relationship between Fe+ ions and equatorial spread F, J. Geophys. Res., 76, 7761-7768, 1971.

Hanson, W. B. and Sanatani, S.: Large Ne gradients below the equatorial F peak, Geophys. Res., 78, 1167-1172, 1971.

Hysell, D. L.: Radar imaging of equatorial F region irregularities with maximum entropy interferometry, Radio Sci., 31, 15671578, 1996.

Hysell, D. L. and Woodman, R. F.: Imaging coherent backscatter radar observations of topside equatorial spread F, Radio Sci., 32, 2309-2320, 1997.

Hysell, D. L. and Burcham, J.: JULIA radar studies of equatorial spread F, J. Geophys. Res., 103, 155-167, 1998.

Hysell, D. L.: Imaging coherent scatter radar studies of bottomside equatorial spread F, J. Atmos. Solar-Terr. Phys., 60, 1109-1122, 1998.

Hysell, D. L.: Imaging coherent scatter radar studies of equatorial spread F. J. Atmos. Terr. Phys., 61, 701-716, 1999.

Hysell, D. L.: An overview and synthesis of plasma irregularities in equatorial spread F, J. Atmos. Solar Terr. Phys., 62, 1037-1056, 2000.

Hysell, D. L. and Kudeki, E.: Collisional shear instability in the equatorial F region ionosphere, J. Geophys. Res., 109, A11301, doi:10.1029/2004JA010636, 2004.

Hysell, D. L., Kudeki, E., and Chau, J. L.: Possible ionospheric preconditioning by shear flow leading to equatorial spread F, Ann. Geophys., 23, 2647-2655, 2005a, http://www.ann-geophys.net/23/2647/2005/.

Hysell, D. L., Larsen, M. F., Swenson, C. M., Barjatya, A., Wheeler, T. F., Sarango, M. F., Woodman, R. F., and Chau, J. L.: Onset conditions for equatorial spread $\mathrm{F}$ determined during EQUIS II, Geophys. Res. Lett., 32, L24104, doi:10.1029/2005GL024743, $2005 b$.

Hysell, D. L. and Chau, J. L.: Optimal aperture synthesis radar imaging, Radio Sci., 41, RS2003, doi:10.1029/2005RS003383, 2006.

Hysell, D. L., Larsen, M. F., Swenson, C. M., Barjatya, A., Wheeler, T. F., Bullett, T. W., Sarango, M. F., Woodman, R. F., Chau, J. L., and Sponseller, D.: Rocket and radar investigation of background electrodynamics and bottom-type scattering layers at the onset of equatorial spread F, Ann. Geophys., 24, 1387-1400, 2006a, http://www.ann-geophys.net/24/1387/2006/.

Hysell, D. L., Larsen, M. F., Swenson, C. M., and Wheeler, T. F.: Shear flow effects at the onset of equatorial spread F, J. Geophys. Res., 111, A11317, doi:10.1029/2006JA011923, 2006b.

Huba, J. D. and Ossakow, S. L.: On the Generation of 3-m Irregularities During Equatorial Spread F by Low-Frequency Drift Waves, J. Geophys. Res., 84, 6697-6700, 1979.

Huba, J. D. and Ossakow, S. L.: On 11-cm Irregularities during Equatorial Spread F, J. Geophys. Res., 86, 829-832, 1981.

Kelley, M. C., Haerendel, G., Carter, D. A., Ecklund, W. L., Carlson, C. W., Hausler, B., and Torbert, R.: Evidence for a Rayleigh- 
Taylor type of instability and upwelling of depleted regions during equatorial spread F, Geophys. Res. Lett., 3, 448-450, 1976.

Kelley, M. C., Larsen, M. F., and La Hoz, C.: Gravity wave interaction of equatorial spread F: A case study, J. Geophys. Res., 86, 9087-9100, 1981.

Kelley, M. C.: The Earth's ionosphere: plasma physics and electrodynamics. International Geophysical Series, 43, Academic Press, San Diego, CA, 1989.

Keskinen, M. J., Ossakow, S. L., and Chamedi, P. K.: Preliminary report of numerical simulations of intermediate wavelength collisional Rayleigh-Taylor instability in equatorial spread F, J. Geophys. Res., 85, 1775-1778, 1980.

Koster J. R. and Wright, R. W.: Scintillation, Spread F, and Transequatorial Scatter, J. Geophys. Res., 65, 2303-2306, 1960.

Kudeki, E. and Bhattacharyya, S.: Postsunset vortex in equatorial F-region plasma drifts and implications for bottomside spread-F, J. Geophys. Res., 104, 28163-28170, 1999.

Kudeki, E., Bhattacharyya, S., and Woodman, R. F.: A new approach in incoherent scatter $\mathrm{F}$ region $E \times B$ drift measurements at Jicamarca, J. Geophys. Res., 104, 28145-28162, 1999.

Kudeki, E., Akgiray, A., Milla, M. A., Chau, J. L., and Hysell, D. L.: Equatorial spread-F initiation: post-sunset vortex, thermospheric winds, gravity waves, J. Atmos. Solar Terr. Phys., 69, 2416-2427, 2007.

Makela, J. J.: A review of imaging low-latitude ionospheric irregularity processes, J. Atmos. Solar-Terr. Phys., 68, 1441-1458, 2006.

Martyn, D. F.: The normal F region of the ionosphere, Proceedings of the IRE, 47, 147-155, 1959.

McClure, J. P., Hanson, W. B., and Hoffman, J. F.: Plasma Bubbles and Irregularities in the Equatorial Ionosphere, J. Geophys. Res., 82, 2650-2656, 1977.

Mendillo, M., Zesta, E., Shodhan, S., Sultan, P. J., Doe, R., Sahai, Y., and Baumgardner, J.: Observations and modeling of the coupled latitude-altitude patterns of equatorial plasma depletions, J. Geophys. Res., 110, A09303, doi:10.1029/2005JA011157, 2005.

Morse, F., A., Edgar, B. C., Koons, H. C., Rice, C. J., Heikkila, W. J., Hoffman, J. H., Tinsley, B. A., Winningham, J. D., Christensen, A. B., Woodman, R. F., Pomalaza, J., and Teixeira, R. N.: Equion an Equatorial Ionospheric Irregularity Experiment, J. Geophys. Res., 82, 578-592, 1977.

Ossakow, S. L.: Spread-F theories, J. Atmos. Terr. Phys., 43, 437452, 1981.

Patra, A. K., Sripathi, S., and Tiwari, D.: Coupling effect of equatorial $\mathrm{F}$ region irregularities on the low latitude $\mathrm{E}$ region instability processes, Geophys. Res. Lett., 31, LI17803, doi:10.1029/2004GL0204486, 2004.

Patra, A. K., Tokoyama, T., Yamamoto, M., Saito, S., Muruyama, T., and Fukao, S.: Disruption of E region echoes observed by the EAR during the development phase of equatorial spread F: A manifestation of electrostatic field coupling, Geophys. Res. Lett., 32, L17104, doi:10.1029/2005GL022868, 2005.

Rastogi, R. G.: On the Equatorial Spread F, Proc. Indian Acad. Sci., 87A, 116-131, 1978.
Rottger, J.: Wave-like structures of large-scale equatorial spread-F irregularities, J. Atmos. Terr. Phys., 35, 1195-1206, 1973.

Scannapieco, A. J. and Ossakow, S. L.: Nonlinear equatorial spread F, Geophys. Res. Lett., 3, 451-454, 1976.

Singh, S., Johnson, F. S., and Power, R. A.: Gravity wave seeding of equatorial plasma bubbles, J. Geophys. Res., 102, 7399-7410, 1997.

Sultan, P.: Linear theory and modelling of the Rayleigh-Taylor instability leading to the occurrence of equatorial spread-F, J. Geophys. Res., 101, 26875-26891, 1996.

Tsunoda, R. T., Barons, M. J., Owen, J., and Towle, D. M.: Altair: an incoherent scatter radar for equatorial spread F studies, Radio Sci., 14, 1111-1119, 1979.

Tsunoda, R.: Magnetic-field-aligned characteristics of plasma bubbles in the nighttime equatorial ionosphere, J. Atmos. Terr. Phys., 42, 743-752, 1980.

Tsunoda, R. and White, B.: On the generation and growth of equatorial backscatter plumes, 1 . Wave structure in the bottomside $\mathrm{F}$ layer, J. Geophys. Res., 86, 3610-3616, 1981.

Tsunoda, R. T., Livingston, R. C., McClure, J. P., and Hanson, W. B: Equatorial plasma bubbles: vertically elongated wedges from the bottomside F layer, J. Geophys. Res., 87, 9171-9180, 1982.

Tsunoda, R. T.: Control of the seasonal and longitudinal occurrence of equatorial scintillations by the longitudinal gradient in integrated E Region Pedersen Conductivity, J. Geophys. Res., 90, 447-456, 1985.

Tsunoda, R. T.: On the enigma of day-to-day variability in equatorial spread F, Geophys. Res. Lett., 32, L08103, doi:10.1029/2005GL022512, 2005.

Valladares, C. E., Villalobos, J., Sheehan, R., and Hagan, M. P.: Latitudinal extension of low-latitude scintillations measured with a network of GPS receivers, Ann. Geophys., 22, 3155-3175, 2004, http://www.ann-geophys.net/22/3155/2004/.

Woodman, R. F.: Irregular Refraction of Artificial Satellite Signals Observed at Ancon, Peru, Advances in Space Research, Proceedings of the First Inter-American Symposium on Space Research, Buenos Aires, Argentina, Pergamon Press LTD, 331-344, 1960.

Woodman, R. F. and Hagfors, T.: Methods for the measurement of vertical ionospheric motions near the magnetic equator by incoherent scattering, J. Geophys. Res., 74, 1205-1212, 1969.

Woodman, R. F. and La Hoz, C.: Radar observations of F region equatorial irregularities. J. Geophys. Res., 81, 5447-5466, 1976.

Woodman, R. F.: Coherent radar imaging: Signal processing and statistical properties, Radio Sci., 32, 2373-2391, 1997.

Woodman, R. F. and Chau, J. L.: Equatorial quasiperiodic echoes from field-aligned irregularities observed over Jicamarca, Geophys. Res. Lett., 28, 207-209, 2001.

Yokoyama, T., Fukao, S., and Yamamoto, M.: Relationship of the onset of equatorial F-region irregularities with the sunset terminator observed with the Equatorial Atmosphere Radar, Geophys. Res. Lett., 31, L24804, doi:10.1029/2004GL021529, 2004.

Zalesak, S. T., Ossakow, S. L., and Chaturvedi, P. K.: Nonlinear equatorial spread $\mathrm{F}$ - the effect of neutral winds and background Pedersen conductivity. J. Geophys. Res., 87, 151-166, 1982. 